\title{
The Generalizability of Online Experiments Conducted During the COVID-19 Pandemic
}

\author{
Kyle Peyton, Gregory A. Huber, and Alexander Coppock * \\ Forthcoming, Journal of Experimental Political Science \\ May 11, 2021
}

\begin{abstract}
The COVID-19 pandemic imposed new constraints on empirical research, and online data collection by social scientists increased. Generalizing from experiments conducted during this period of persistent crisis may be challenging due to changes in how participants respond to treatments or the composition of online samples. We investigate the generalizability of COVID-era survey experiments with 33 replications of 12 pre-pandemic designs, fielded across 13 quota samples of Americans between March and July of 2020. We find strong evidence that pre-pandemic experiments replicate in terms of sign and significance, but at somewhat reduced magnitudes. Indirect evidence suggests an increased share of inattentive subjects on online platforms during this period, which may have contributed to smaller estimated treatment effects. Overall, we conclude that the pandemic does not pose a fundamental threat to the generalizability of online experiments to other time periods.
\end{abstract}

\footnotetext{
${ }^{*}$ Kyle Peyton is Postdoctoral Fellow in Law and Social Science, Yale Law School (kyle.peyton@yale.edu); Gregory A. Huber is Forst Family Professor of Political Science, Yale University (gregory.huber@yale.edu); Alexander Coppock is Assistant Professor of Political Science, Yale University (alex.coppock@yale.edu). Thanks to the Center for the Study of American Politics and the Institution for Social and Policy Studies for research support, to Peter Aronow, Josh Kalla, Lilla Orr, John Ternovski, and Baobao Zhang for helpful comments and feedback, to Ethan Porter for sharing replication materials, to Antonio Arechar, Matt Graham, David Rand, Patrick Tucker, Chloe Wittenberg, and Baobao Zhang for sharing pre-COVID survey data. We thank Allison St. Martin and Patrick Comer of Lucid for enlightening conversations and providing aggregate data on purchases made by academic clients. Previous versions of the manuscript were presented and benefited from feedback at Université de Montréal, Australian National University, UMass Amherst, and York University. This research was approved by the Yale University Institutional Review Board (Protocol number 1312013102).
} 
During the COVID-19 pandemic, social scientists across the globe have been forced to abandon or postpone research projects that require face-to-face interaction, travel, or even simply leaving the house. Not surprisingly, the use of online surveys - a relatively low cost form of empirical research that does not require in-person data collection - expanded dramatically during the COVID-19 pandemic. For example, purchases by academic clients on Lucid - a widely used marketplace for survey respondents - nearly tripled between 2019 and 2020. Amid this boom in online research activity, concerns have been raised about the external validity of experiments conducted during this period (e.g., IJzerman et al., 2020; Rosenfeld et al., forthcoming). Here we investigate whether extrapolations from studies conducted during the pandemic to other time periods will be misleading.

Empirically demonstrating that experiments conducted during the COVID era do or do not generalize to other times is straightforward in principle. Once the pandemic has passed and the social, economic, and political aftershocks have dissipated, replications of COVIDera studies can settle the question of whether those results generalize to the post-crisis period. Unfortunately, it may be a while before normal times fully resume. In this paper, we take up a closely-related question that we can answer much sooner: do experiments conducted prior to the pandemic generalize to COVID times?

We provide an answer using 33 replications fielded during the onset of the COVID-19 pandemic of 12 previously-published survey experiments. Consistent with the findings from recent replication attempts on different samples, we find strong evidence of correspondence. Our COVID-era replication estimates nearly always agree with pre-COVID estimates in terms of sign and significance, but are somewhat smaller in magnitude at an average of $73 \%$ of the pre-COVID effect size. We argue that this pattern may be explained by lower levels of attentiveness among online survey respondents during the initial onset of the pandemic. Overall, the replication estimates presented here should mitigate concerns that results from survey experiments conducted during the pandemic will not generalize to other times and contexts.

\section{Background}

A common framework for understanding how results generalize from one sample to a target population is the "UTOS" framework, which stands for Units, Treatments, Outcomes, and Settings (Cronbach and Shapiro, 1982). The framework predicts that results will exhibit greater correspondence (or generalizability or transportability) between a sample and the 
target population with increasing similarity of their UTOS components. A decade's worth of studies in political science have invoked the UTOS framework to argue that survey experiments conducted with online samples generalize to the U.S. national population (Berinsky, Huber and Lenz, 2012; Mullinix et al., 2015; Coppock, 2019; Coppock, Leeper and Mullinix, 2018; Coppock and McClellan, 2019). Recent work by Findley, Kikuta and Denly (2020) elaborates the UTOS framework to differentiate the time dimension from other features of settings, pointing out that the mechanisms that link treatments to outcomes may change over time. Munger (2020) describes growing concerns about generalizability across time as a question of "temporal validity."

The COVID-19 pandemic led to changes in at least two important factors relevant to online survey experiments. First, nearly everyone experienced massive disruptions to their daily lives. These changes may have affected how online survey participants respond to treatment. For example, Americans may have been in a heightened state of anxiety due to a variety of factors, including an unmitigated public health crisis, rising economic insecurity, and chronic political instability. Anxiety and other emotional state variables have been shown to affect information processing (e.g., Gadarian and Albertson, 2014), willingness to dissent (e.g., Young, 2019), and to condition effects of other treatments (e.g., Valentino et al., 2009).

Another important factor is that the pandemic altered, or was coincident with, changes in the composition of markets for online survey respondents. Arechar and Rand (2020), for example, find that an influx of new workers to the Amazon Mechanical Turk (MTurk) platform in March 2020 led to samples that were less attentive, but more demographically diverse and representative of the U.S. population. On the Lucid platform, the demand for survey respondents in 2020 increased by roughly 40\% among commercial clients, and by $200 \%$ among academic clients (see Figure 1). In the immediate wake of the pandemic, Lucid suppliers - who furnish survey respondents for both academic and commercial clients - struggled to recruit enough new participants to meet this increased demand. ${ }^{1}$ Consistent with the possibility that suppliers attempted to meet this increased demand with relatively lower-quality respondents, Aronow et al. (2020) find that respondent attentiveness on Lucid was declining over the period March-July 2020. This decline in attentiveness may also have reflected changes in the types of people who were previously attentive becoming less attentive because of COVID-19 related disruptions.

\footnotetext{
${ }^{1}$ Conversation with Lucid representatives on 18 March 2021. According to Lucid, supply gradually increased over the summer of 2020, just as demand for survey responses intensified in the lead up to the 2020 election.
} 
Figure 1: Total weekly survey responses completed and sold to academic buyers by Lucid between January 2019 and March 2021

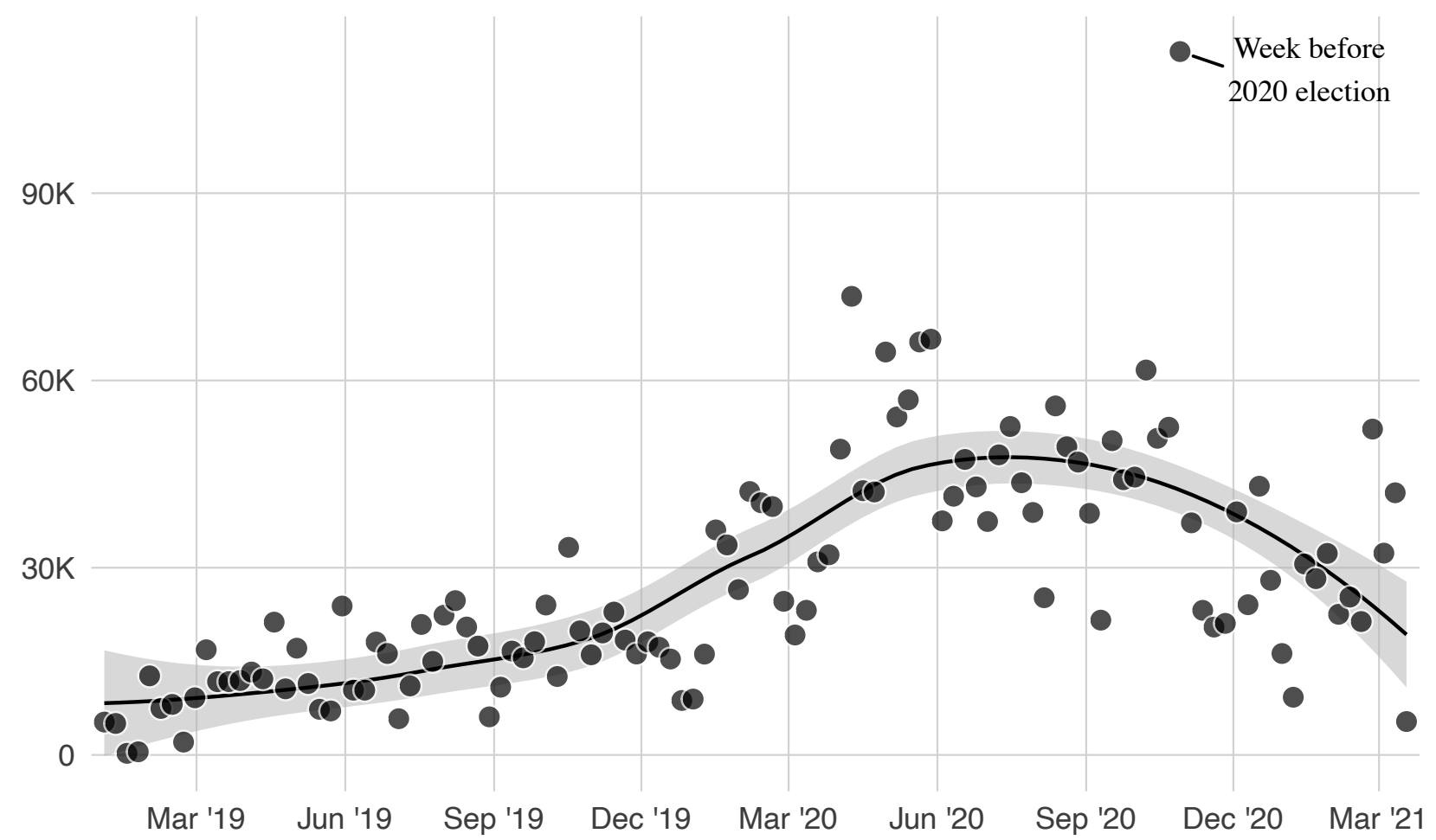

Notes: Points denote weekly survey responses completed and sold to academic customers by Lucid (in thousands). Dark lines and 95\% confidence bands from loess smoother. The WHO declared COVID-19 a pandemic on 11 March 2020. Proprietary data provided by Lucid cover the period 1 January 2019 - 18 March 2021. In 2019 Lucid sold 729,284 completed survey responses to academic buyers, compared with $2,185,387$ in 2020 .

\subsection{Design}

Given the proliferation of online research conducted during the COVID-19 pandemic, we can articulate concerns about external validity as an empirical question: would the results from an experiment conducted during the pandemic have been different if the experiment were instead conducted in a different period?

Between March and July 2020, we recruited weekly samples of approximately 1,000 U.S. based participants via Lucid. Each survey was between 10 and 15 minutes long (median duration: 12.8 minutes) and was structured in discrete 3-5 minute modules. Lucid collects demographic information from all respondents before they enter any particular survey, enabling the construction of quota samples that approximate U.S. census margins (Coppock 
and McClellan, 2019). Like previous investigations on the suitability of convenience samples for academic research, we focus on survey experiments in particular. Using online convenience samples for descriptive work is generally inadvisable because the samples may differ from target populations in both observable and unobservable ways. ${ }^{2}$ However, for theoretical settings in which an average treatment effect among a diverse (but nevertheless unrepresentative) sample would be informative, survey experiments with online convenience samples are an effective and widely-used tool.

\subsubsection{Selection criteria}

We conducted 33 replications across 12 unique studies, chosen based on the following criteria:

1. Suitable for online survey environment. All replications were administered through a web browser using Qualtrics survey software, and the original studies must have been fielded in a similar format.

2. Length of study. Time constraints ruled out studies that could not be administered in a survey module of 3-5 minutes.

3. Design transparency. The original study design and outcome measures were clearly described in the published manuscript or supporting appendix.

4. Design complexity and effect size. Sample size constraints ruled out some two-arm studies with small effect sizes, as well as more complex studies with elaborate factorial designs.

5. Theoretical and political importance. The studies all considered theoretically important questions, with many being published in top journals.

These criteria are similar to those used in other meta-scientific replication projects (e.g., Klein et al., 2014, 2018). We also aimed for a mix of classic and contemporary studies, and for coverage across political science sub-fields. Our set of studies is not a random sample of all survey experiments conducted by social and political scientists, but they do cover a wide range of designs. The full set of studies is listed in Table 1. We conducted at least one direct replication of each study. Modified versions of studies 2, 3, 5, and 7 were also replicated using COVID-specific content. A description of each modified version and their corresponding replication estimates are provided in Online Appendix Section A.

\footnotetext{
${ }^{2}$ Balance on the demographic marginal distributions does not imply balance on the joint distributions. Moreover, because these are not probability samples, the degree of imbalance on the distribution of unobserved attributes is unknown.
} 
We categorize each replication as a "success" if the COVID-era estimate(s) are correctly signed and statistically distinguishable from zero. For studies with "null" results (studies 11-12), replication was declared successful if estimate(s) were indistinguishable from zero and their pre-COVID benchmarks. A replication "failure" occurs when estimate(s) are incorrectly signed, regardless of whether they are distinguishable from zero. For studies with multiple treatment arms/outcomes, we concluded replication was successful if the preponderance of evidence supports success. In one case, the preponderance of evidence was ambiguous and we concluded the replication attempt was a partial success. Additional details are available in Online Appendix Section A.

Table 1: Summary of thirty-three replications conducted across twelve original studies

\begin{tabular}{|c|c|c|c|}
\hline Original study & Design & Replications & Success \\
\hline $\begin{array}{l}\text { 1. Russian reporters and American news } \\
\text { (Hyman \& Sheatsley, 1950) }\end{array}$ & Two-arm & Week 3 & Yes \\
\hline $\begin{array}{l}\text { 2. Effect of framing on decision making } \\
\text { (Tversky \& Kaheneman, 1981) }\end{array}$ & Two-arm & Week 7 & Yes \\
\hline $\begin{array}{l}\text { 3. Gain versus loss framing } \\
\text { (Tversky \& Kaheneman, 1981) }\end{array}$ & Two-arm & Weeks $1,3,7,8,13$ & Yes \\
\hline $\begin{array}{l}\text { 4. Welfare versus aid to the poor } \\
\text { (Smith, 1987) }\end{array}$ & Two-arm & Weeks 1-9, 11-13 & Yes \\
\hline $\begin{array}{l}\text { 5. Gain vs. loss framing + party endorsements } \\
\text { (Druckman, 2001) }\end{array}$ & Six-arm & Weeks $7,8,13$ & Yes \\
\hline $\begin{array}{l}\text { 6. Foreign aid misperceptions } \\
\text { (Gilens, 2001) }\end{array}$ & Two-arm & Week 3 & No \\
\hline $\begin{array}{l}\text { 7. Perceived intentionality for side effects } \\
\text { (Knobe, 2003) }\end{array}$ & Two-arm & Week 7 & Yes \\
\hline $\begin{array}{l}\text { 8. Atomic aversion } \\
\text { (Press, Sagan, \& Valentino, 2013) }\end{array}$ & Five-arm & Weeks $5,6,13$ & Partial \\
\hline $\begin{array}{l}\text { 9. Attitudes towards immigrants } \\
\text { (Hainmueller \& Hopkins, 2015) }\end{array}$ & Factorial (conjoint) & Week 8 & Yes \\
\hline $\begin{array}{l}\text { 10. Fake news corrections } \\
\text { (Porter, Wood, \& Kirby, 2018) }\end{array}$ & Mixed factorial $(2 \times 6)$ & Week 4 & Yes \\
\hline $\begin{array}{l}\text { 11. Inequality and system justification } \\
\text { (Trump \& White, 2018) }\end{array}$ & Two-arm & Week 2 & Yes \\
\hline $\begin{array}{l}\text { 12. Trust in government and redistribution } \\
\text { (Peyton, 2020) }\end{array}$ & Three-arm & Week 9 & Yes \\
\hline
\end{tabular}




\section{Results}

We were able to obtain the original effect size(s) for each of the 12 pre-COVID studies listed in Table 1, and at least one pre-COVID replication estimate of the original effect size(s) for 7 of these studies. In total, we obtained 89 pre-COVID estimates and 101 replication estimates. ${ }^{3}$ A detailed description of each study, their pre-COVID estimates, and the individual replication estimates that we obtained are provided in Section A of the Online Appendix. Here we provide an overall summary for each study by comparing the pooled estimates from our COVID-era replications with the pooled estimates from the pre-COVID benchmarks (original estimates and any pre-COVID replications).

For each study, we calculate summary effect sizes for each treatment-outcome pair using a precision-weighted average, with weights based on the reciprocal of the variance. We can then compare the pre-COVID and replication estimates by taking the differences between their summary effect size estimates. For studies with one outcome and a simple experimental design, we compute a single difference; and for studies with multiple outcomes and/or treatments, we compute a difference for each unique treatment-outcome pair. Except for the conjoint experiment, all within-study estimates (binary and ordinal) are standardized using Glass's $\Delta$, which scales outcomes by the standard deviation in the control group (Glass, 1976).

Across the 12 studies, we obtained 138 summary effect size estimates - 82 for the conjoint studies and 56 for the remaining studies. For the non-conjoint studies, Figure 2 compares the 28 estimated summary effects from the pre-COVID studies (horizontal axis) with their 28 replications (vertical axis). All replication summary estimates were smaller in magnitude than their pre-COVID estimates, with 24 of 28 signed in the same direction. Of the 24 correctly signed estimates, 10 were significantly smaller in replication. Of the 4 incorrectly signed estimates, 3 were significantly different - the foreign aid misperceptions study and 2 of 6 estimates from the atomic aversion study. Figure 3 plots the analogous information for the 41 conjoint estimates and their 41 replications, all of which are signed in the same direction. Of these, 35 of 41 were smaller in replication (6 statistically significant differences) and 6 of 41 were larger in replication (1 of 6 significant differences).

Pooling across all 65 of 69 correctly signed pairs presented in Figures 2-3, the replication

\footnotetext{
${ }^{3}$ The General Social Survey (GSS) has administered the welfare versus aid to the poor experiment 20 times between 1986 and 2018. If all GSS estimates are included, we have 108 pre-COVID estimates in total. Given the variation in question wordings and survey modes across time, we use the 1986 GSS experiment as our "original" estimate, and take the first two replications conducted on online samples by Huber and Paris (2013) as our pre-COVID replication estimates.
} 
estimates were, on average, $73 \%$ as large as the pre-COVID estimates. The 41 correctly signed estimates from the conjoint replication were, on average, $87 \%$ as large as the original. The 24 of 28 correctly signed estimates from the other replications were, on average, $49 \%$ as large as the pre-COVID summary effect sizes.

When compared with other replication efforts, the correspondence for the conjoint experiment is high whereas the correspondence for the others is modest. For example, in one of the earliest large-scale replications, Open Science Collaboration (2015) replicated 100 experiments from three top psychology journals and found that about $40 \%$ replicated and effect sizes were, on average, about half the magnitude of the original effects. In economics, Camerer et al. (2016) replicated 18 survey and lab-based experiments and found effect sizes in the 11 studies that successfully replicated were approximately $66 \%$ of the original. Coppock (2019) replicated 40 treatment effect estimates from 12 survey experiments, finding that the median MTurk estimate was $66 \%$ of the original effect size.

Common explanations for somewhat diminished effect sizes in replication studies include imperfect adherence to the original experimental protocol on the part of replicators and publication pressures that systematically select for larger effect sizes in original study journal submissions. These mechanisms may be operative here as well. At the same time, we do not think they are the main reasons for the smaller effect sizes we find because the experimental protocols are relatively straightforward and the bulk of these studies had been successfully replicated by others before us with larger effect sizes than we find. Prior replications of studies 7 and 8, for example, have found effect sizes at least as large as the original (see Klein et al., 2018; Aronow, Baron and Pinson, 2019). 
Figure 2: Comparison of 28 summary effect sizes across 11 studies (conjoint excluded)

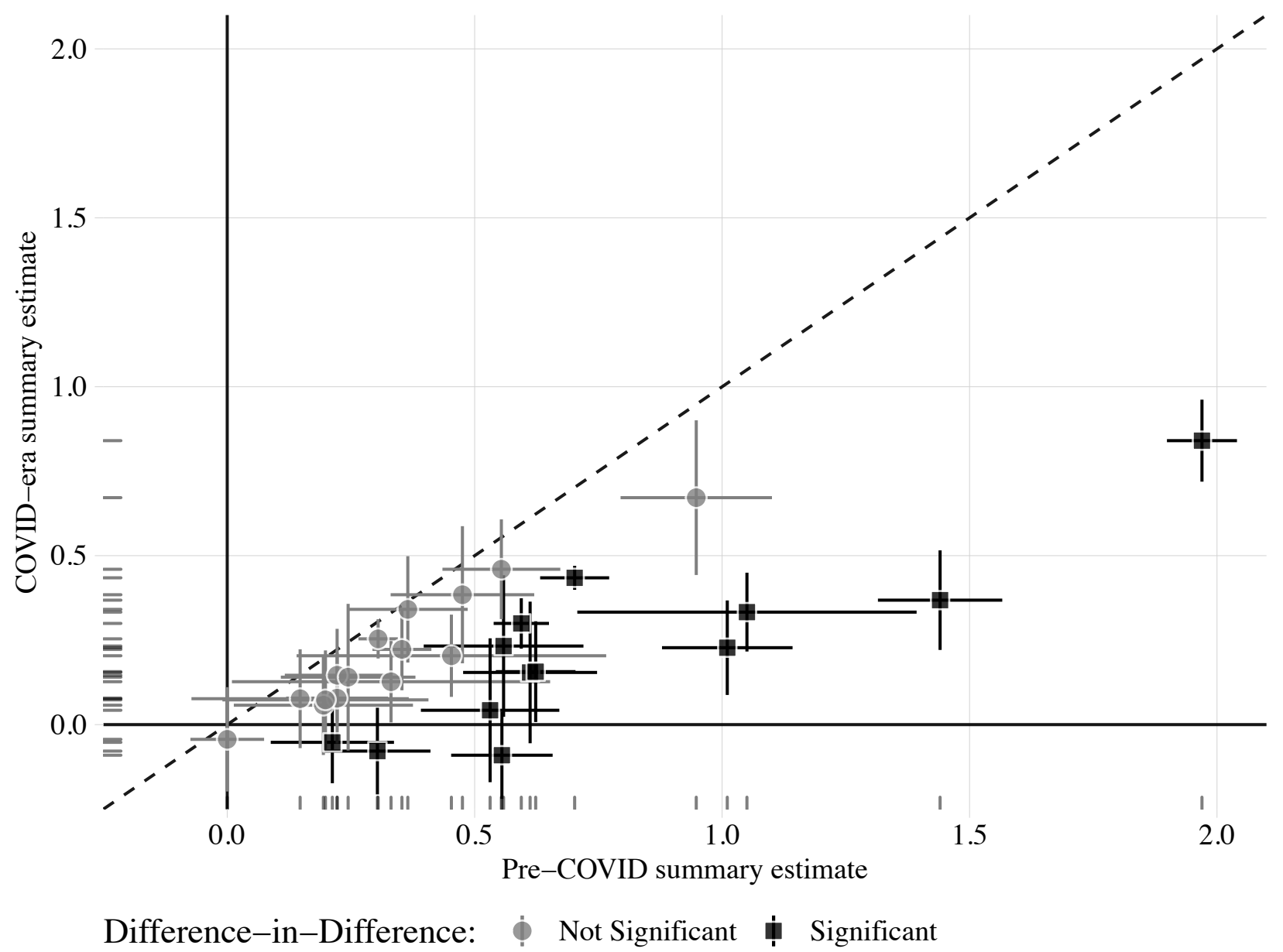

Notes: Estimated summary effect sizes and 95\% confidence intervals from pre-COVID experiments (horizontal axis) and COVID-era replications (vertical axis). 13 of 28 replication estimates were significantly different from their pre-COVID benchmark at $p<0.05$. 
Figure 3: Comparison of 41 summary effect sizes in conjoint experiments

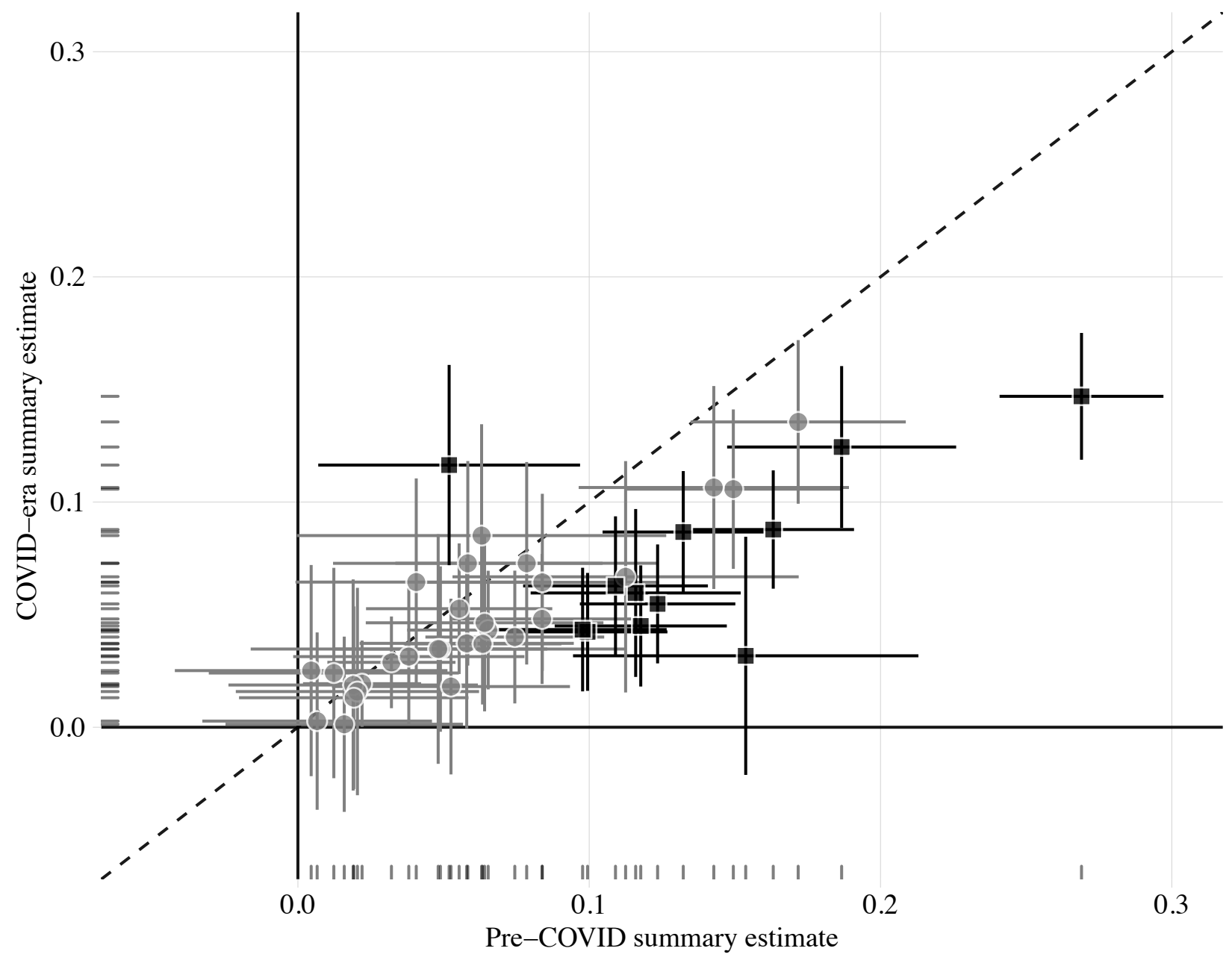

\section{Difference-in-Difference: Not Significant Significant}

Notes: Estimated summary effect sizes and 95\% confidence intervals from pre-COVID experiments (horizontal axis) and COVID-era replications (vertical axis). 7 of 41 replication estimates were significantly different from their pre-COVID benchmark at $p<0.05$. 


\section{Can inattention explain attenuated replication esti- mations?}

In this section, we consider declining attentiveness among survey respondents during the COVID-era as a potential explanation for attenuated treatment effect estimates. This decline in attention - as measured by increased failure rates on Attention Check Questions (ACQs) in Aronow et al. (2020) and Arechar and Rand (2020) - might reflect a genuine decline in attention among online survey respondents, underlying changes in the types of people who participate in online research, or both. Although we cannot distinguish among these possibilities, prior work has demonstrated that inattention leads to measurement error in self-administered surveys. Berinsky, Margolis and Sances (2014), for example, replicated the gain versus loss framing experiment from Tversky and Kahneman (1981) and found an estimated treatment effect of approximately zero among respondents that failed a pretreatment attention screener.

Measurement error induced by inattention is nonrandom, so the correlations across covariates and survey outcomes can be overstated or understated in descriptive survey work. In the experimental setting, however, inattention generates a form of treatment noncompliance. To fix ideas, suppose that an experimental sample can be partitioned into two types of individuals: the "attentive" and the "inattentive". Imagine the average effect among the attentive is positive and the average effect among the inattentive is zero, because these

subjects do not engage with treatment and are therefore unaffected by it. The Average Treatment Effect (ATE) in the full sample is therefore a weighted average of two quantities: the average effect among the attentive, and the average effect among the inattentive. The ATE estimated for the full sample will therefore be closer to zero than the average effect among the attentive. Estimates for the overall ATE attenuate towards zero as the share of inattentive subjects in the sample grows.

For ease of exposition, we have dichotomized subjects as attentive or inattentive, but respondent attention in online surveys is both continuous and dynamic (Berinsky, Margolis and Sances, 2016; Berinsky et al., 2019). It is continuous because subjects may be more or less attentive and it is dynamic because attention may change during a survey. Accounting for these subtleties is important, but the core argument is that estimated treatment effects will attenuate towards zero as inattention increases. If subjects can be classified as one type or the other, then we expect smaller effects where the share of the inattentive is higher. If subjects pay partial attention, we expect that estimated treatment effects among the 
partially attentive will be smaller what they would have been had these subjects paid closer attention. Given that the proportion of inattentive subjects in online samples appears to have increased during the period in which our replications were conducted (see Aronow et al., 2020; Arechar and Rand, 2020), this may partly explain why our replication estimates are smaller when compared to pre-COVID estimates.

We can examine this possibility by estimating effects among the attentive and inattentive separately. The most straightforward approach is to include pre-treatment ACQs (see Oppenheimer, Meyvis and Davidenko, 2009; Paolacci et al., 2010). ${ }^{4}$ However, only two of our surveys included ACQs, so we consider alternative approaches as well.

A second approach is to rely on individual-level meta-data. One possibility is that increased inattention during the COVID-era stems from an increasing proportion of respondents arriving to the survey environment from mobile games. ${ }^{5}$ The "User Agent" string captured from the end user's browser by online survey software like Qualtrics provides detailed information about how respondents arrive at the survey. We offer two approaches for classifying respondents as inattentive on the basis of this information: 1) if they come from a web-application rather than a web-browser; 2) if they come from a mobile phone rather than a tablet or desktop.

Applying this approach across the 13 surveys used for the replication studies, we estimate that the proportion of participants coming from web-applications (rather than internet browsers) ranged from 0.19 to 0.61 , and the proportion of participants coming from mobiles (rather than desktops or tablets) ranged from 0.31 to 0.73. Based on an additional sample of 63,245 respondents obtained from Lucid surveys fielded prior to March 2020, we observe that the proportion of participants from both web-applications and mobiles was trending upward prior to the COVID-19 outbreak. In the 2020 surveys, approximately $41 \%$ of respondents came from web applications ( $56 \%$ from mobiles), an increase from $33 \%$ ( $56 \%$ from mobiles) in 2019 and just 13\% (33\% from mobiles) in 2018. These results, reported in Figure 4, are consistent with the declining data quality documented by Aronow et al. (2020).

\footnotetext{
${ }^{4}$ Kane, Velez and Barabas (2020) suggest including "mock vignettes" which can be viewed as a taskspecific ACQ. Berinsky, Margolis and Sances (2014); Berinsky et al. (2019) urge researchers to use multiple ACQs and classify subjects based on different levels of attentiveness.

${ }^{5}$ In a separate Lucid survey fielded on October 29th we filtered out respondents who failed an ACQ at the beginning of the survey (the "Easy" ACQ from Table 2). Immediately prior to this, we asked respondents to self-report whether they were recruited to the survey from a game, and $51 \%$ reported they were. We observed substantial differences in pass rates: among those coming from an online game, only $38 \%$ passed the ACQ, versus $82 \%$ of those not coming from a game. Interviews with Lucid representatives in 2021 suggest suppliers that have since been removed from the platform were providing these respondents from mobile games.
} 
Figure 4: Respondents from mobile devices and web applications from Jun 2018 to Jul 2020

Respondents from mobile phones

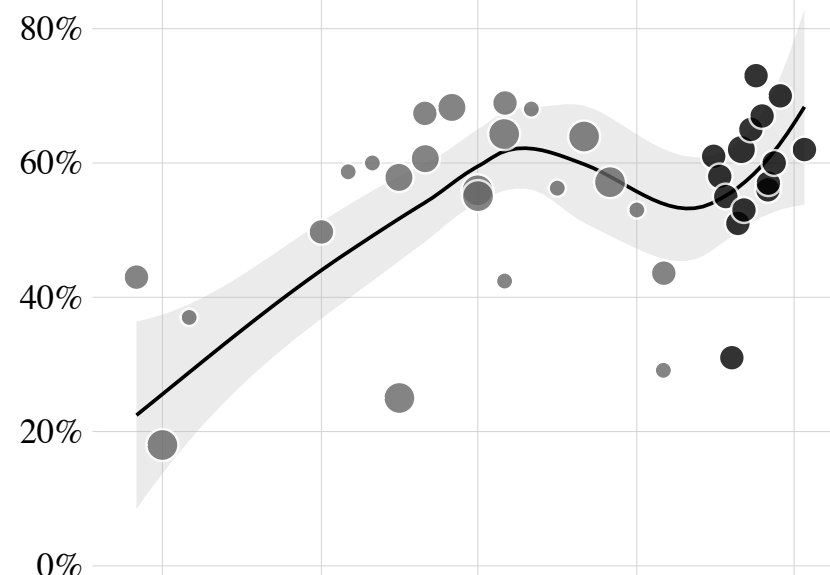

\section{Respondents from web applications}

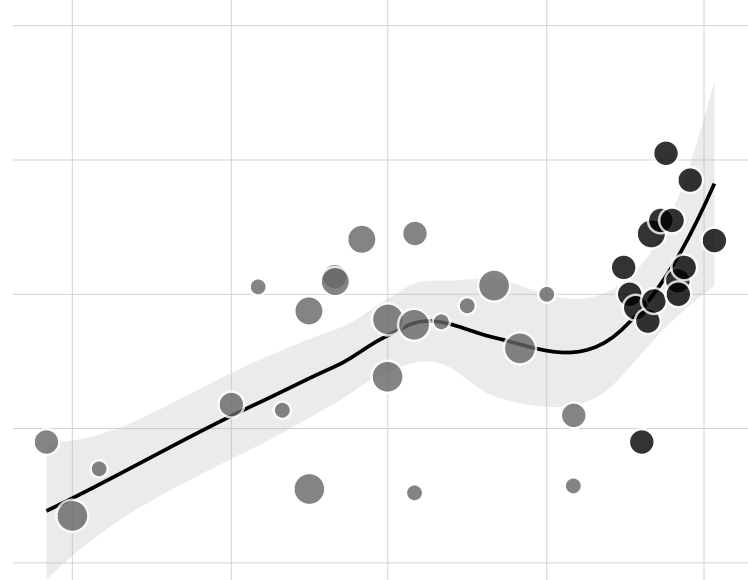
Jul '18 Jan '19 Jul '19
Jan '20
Jul '20 Jul '18
Jan '19
Sample size: $\bigcirc<1,000 \bigcirc 1,000-2,000 \bigcirc 2,000-3,000 \bigcirc 3,000+$
Pre-COVID sample: • No $\bullet$ Yes

Jul '19 Jan '20

Jul '20

Notes: Points denote percentage of respondents in each Lucid survey, sized in proportion to sample size. Dark lines and 95\% confidence bands from loess smoother. Estimates are based on individual-level browser meta-data and come from a combination of surveys conducted by the authors and UserAgent data shared by Antonio Arechar, Matt Graham, Patrick Tucker, Chloe Wittenberg, and Baobao Zhang.

Pooling across the 13 surveys used for our replication studies, $97 \%$ of participants from web-applications were also on mobile devices, and $72 \%$ on mobile devices arrived from webapplications. Those from web-applications spent approximately 7 minutes less time completing surveys than those from web browsers, who spent an average of 21.5 minutes. Respondents from mobile devices spent roughly 6 minutes less time completing surveys than subjects from non-mobile devices. Additionally, in two of the 13 surveys we included ACQs of varying difficulty and found those on web-applications (or mobiles) were significantly less likely to pass the ACQs, compared to those coming from browsers (or non-mobiles). These results are reported in Table 2. 
Table 2: ACQ pass rates by attentiveness and level of difficulty

\begin{tabular}{llll|lll}
\hline Difficulty & Browser & Web-App & Difference & Non-Mobile & Mobile & Difference \\
\hline Easy & $0.66(0.02)$ & $0.55(0.02)$ & $0.11(0.03)^{*}$ & $0.63(0.02)$ & $0.58(0.02)$ & $0.05(0.03)$ \\
Medium & $0.53(0.02)$ & $0.37(0.02)$ & $0.16(0.03)^{*}$ & $0.50(0.02)$ & $0.42(0.02)$ & $0.08(0.03)^{*}$ \\
Hard & $0.22(0.02)$ & $0.15(0.01)$ & $0.07(0.02)^{*}$ & $0.24(0.02)$ & $0.16(0.01)$ & $0.08(0.02)^{*}$ \\
\hline
\end{tabular}

Notes: The "Easy" and "Medium" questions were novel ACQs that subjects completed after reading a short news article (see Fig. C.9-C.10). The "Hard" ACQ comes from Peyton (2020) and was included with the direct replication of the original study in Week 9. This ACQ, is analogous to an "Instructional Manipulation Check" (see Oppenheimer, Meyvis and Davidenko, 2009) and was passed by $87 \%$ of respondents in the original study (see Fig. C.11). Standard errors in parentheses. $p<0.05^{*}$.

While most of our surveys did not include pre-treatment ACQs, we did include one in the direct replication of Peyton (2020), allowing for a direct comparison of estimates among the attentive using both the ACQ and metadata approaches. Figure 5 shows that regardless of the approach used to classify subjects as attentive, estimated treatment effects among the attentive are positive and significant. Among those who passed the ACQ, the average effect of treatment was 0.39 (SE: 0.15). Among those coming from browsers, the estimate was 0.33 (SE: 0.10), and among non-mobile users it was 0.52 (SE: 0.12). Among those who failed the ACQ (estimate: 0.08, SE: 0.09), came from a web-application (estimate: -0.03, SE: 0.12), or used a mobile phone (estimate: -0.04, SE: 0.10), estimated treatment effects were all close to zero and nonsignificant. 
Figure 5: Reanalysis of estimated treatment effects on trust in government for Peyton (2020) replication

\section{Original experiments:}

MTurk sample, Jun 2014

Qualtrics sample, Sep 2014

MTurk sample, Mar 2017

\section{Lucid replication:}

Full Sample

Attentive: Passed ACQ

Inattentive: Failed ACQ

Attentive: Internet browser

Inattentive: Web-Application

Attentive: Non-mobile device

Inattentive: Mobile device

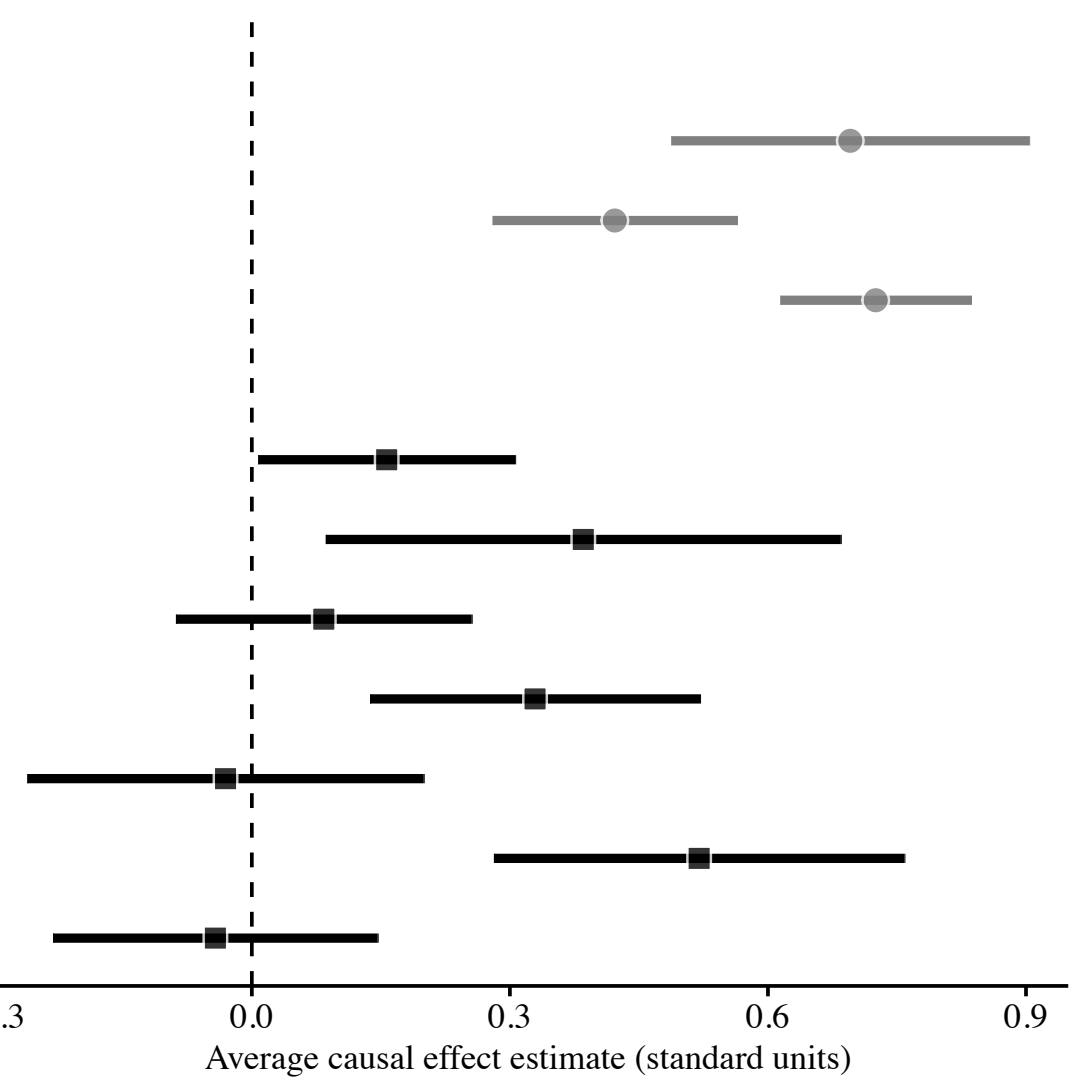

Notes: Estimates for the Lucid replication, fielded in May 2020, are presented for the full sample, and each sub-group partition created by three different methods for classifying attentive v. inattentive respondents. Pairwise correlations between these methods: 0.67 for Non-mobile and Browsers, 0.10 for Non-mobiles and ACQ pass, and 0.09 for Browsers and ACQ pass. Estimates for the original experiments come from the replication archive for Peyton (2020) at https://doi.org/10.7910/DVN/L3NT6P. The Qualtrics sample was a quota sample that, like our Lucid replication sample, approximates U.S. census margins on respondents demographics. In the MTurk sample from March 2017, Peyton (2020) Appendix S5.8 reports $87 \%$ of respondents passed their pre-treatment ACQ. In our direct replication, $19 \%$ of respondents passed this same ACQ.

A final approach is to reason about the plausible scope for inattention to explain the attenuated treatment effects we find. If we assume that treatments do not affect outcomes among inattentive respondents, we can recover the effect among attentive respondents by dividing the overall estimates by the proportion of attentive respondents. If this assumption is incorrect, either because treatment effects among the inattentive are non-zero or because certain treatments affect attention, then this adjustment could be biased upward or downward. 
Pooling across all replication studies, we find effect sizes(s) were, on average, $73 \%$ their pre-COVID magnitude. If $73 \%$ of respondents were attentive and $26 \%$ inattentive, our replication estimate would, on average, match our pre-COVID benchmarks. Empirical estimates of inattention suggest this rate is not unreasonable. Aronow et al. (2020) report that approximately $70 \%$ of respondents passed ACQs in surveys fielded between March and July 2020. Dividing each of the COVID-era summary estimates that we obtained by 0.70 yields an estimated replication correspondence of $104 \%$ the pre-COVID effect sizes.

Of course, it is unlikely the case that pre-COVID samples were entirely attentive, in which case we would also need to adjust those estimates upward, implying that even accounting for growing inattention cannot fully explain smaller effect sizes during COVID-19. We emphasize that this does not constitute proof that inattention explains the attenuated effect sizes across our replications. Instead, we offer it as a partial explanation that is consistent with the evidence provided in Figure 5 and the broader trend of declining attentiveness among online survey respondents during the early onset of the pandemic.

\section{Discussion}

Our goal in this replication study was to understand whether online experiments conducted during the COVID-19 pandemic would generalize to other periods. We investigated by conducting 33 replications during the early COVID era of 12 published experimental studies. Overall, we find strong evidence that these experiments replicated in terms of sign and significance, but at reduced magnitudes. Because these pre-COVID experiments were, with one exception, successfully replicated we infer that the pandemic does not pose a fundamental threat to the generalizability of results from online experiments conducted during this period.

We considered two ways in which treatment effects might have been different during this period. First, we considered the possibility that the pandemic and the significant changes it brought to all aspects of daily life changed individuals' attitudes and patterns of information processing. If so, then the same experiment conducted on otherwise similar groups of people (online survey takers) before and during the pandemic might yield fundamentally different results. We do not find support for this idea. Second, it's possible that the pandemic changed, or occurred alongside changes to, the online survey respondent pool. Decreased attentiveness, as documented by others (Arechar and Rand, 2020; Aronow et al., 2020), provides some indication that the composition of online samples changed, at least during the early onset of the pandemic. 
Using a variety of means - attention check questions, subject meta-data, and external estimates of attentiveness - we conclude that declining attentiveness in online samples may at least partly explain why the replication estimates presented here are, on average, smaller than pre-COVID benchmarks. Inattention can reduce respondents' compliance with experimental stimuli (shrinking average treatment effect estimates towards zero) and it adds nonrandom measurement error to outcome variables (decreasing the precision of treatment effect estimates). One important implication is that rising inattention in online samples can decrease the false positive rate at the cost of increasing the false negative rate. That is, inattention can bias estimates in the conservative direction such that small but substantively important treatment effects may be harder to detect.

Ultimately, answering the empirical question of whether treatment effects are equivalent for those who are attentive and those who are not requires a design that induces the inattentive to pay attention. Unfortunately, prior research has shown that inducing attentiveness among inattentive subjects is difficult (Berinsky, Margolis and Sances, 2016). We experienced these same challenges in our own work. In a separate Lucid study, we attempted to induce attentiveness by randomly assigning some respondents who failed an initial attention check to a condition in which we told them that they failed and gave them a second chance to pass. Only 17 of the 410 subjects (4\%) in this treatment group passed when specifically reminded to re-read the prompt carefully because they had missed something, suggesting it is still difficult to induce attentiveness.

We therefore recommend that researchers concerned about inattention in online samples include pre-treatment attention checks (Berinsky, Margolis and Sances, 2014; Permut, Fisher and Oppenheimer, 2019). Post-treatment attention checks can induce bias (Montgomery, Nyhan and Torres, 2018; Aronow, Baron and Pinson, 2019), but pre-treatment attention checks allow researchers to either terminate the interview without paying for inattentive respondents at the design stage, or retain them and use attention checks to compare estimates between attentive and inattentive subgroups at the analysis stage. We caution, however, that the former strategy necessarily restricts inferences to the sub-group of respondents that passed an attention check task, and these respondents may differ from those that failed on other dimensions as well (see Berinsky, Margolis and Sances, 2014, Online Appendix C).

In sum, we conclude that the pandemic does not pose a fundamental threat to the generalizability of online experiments, provided respondents pay attention to treatment content and outcome questions. People still prefer riskier options for responding to unusual disease outbreaks when in a loss frame - even when the real-world analogue could hardly be more 
salient. People still believe misinformation, but can be corrected with fact checks. People still have preferences over immigrants that can be measured via a conjoint experiment. People still prefer funding "aid to the poor" to "welfare." Even in extraordinary times, we find mostly ordinary responses to treatment in online samples. 


\section{References}

Arechar, Antonio A and David Rand. 2020. "Turking in the time of COVID." PsyArXiv .

Aronow, Peter M, Jonathon Baron and Lauren Pinson. 2019. "A note on dropping experimental subjects who fail a manipulation check." Political Analysis 27(4):572-589.

Aronow, Peter Michael, Joshua Kalla, Lilla Orr and John Ternovski. 2020. "Evidence of Rising Rates of Inattentiveness on Lucid in 2020." SocArXiv .

Berinsky, Adam J, Gregory A Huber and Gabriel S Lenz. 2012. "Evaluating online labor markets for experimental research: Amazon.com's Mechanical Turk." Political analysis 20(3):351-368.

Berinsky, Adam J, Michele F Margolis and Michael W Sances. 2014. "Separating the shirkers from the workers? Making sure respondents pay attention on self-administered surveys." American Journal of Political Science 58(3):739-753.

Berinsky, Adam J, Michele F Margolis and Michael W Sances. 2016. "Can we turn shirkers into workers?" Journal of Experimental Social Psychology 66:20-28.

Berinsky, Adam J, Michele F Margolis, Michael W Sances and Christopher Warshaw. 2019. "Using screeners to measure respondent attention on self-administered surveys: Which items and how many?" Political Science Research and Methods pp. 1-8.

Camerer, Colin F, Anna Dreber, Eskil Forsell, Teck-Hua Ho, Jürgen Huber, Magnus Johannesson, Michael Kirchler, Johan Almenberg, Adam Altmejd, Taizan Chan et al. 2016. "Evaluating replicability of laboratory experiments in economics." Science 351(6280):1433-1436.

Coppock, Alexander. 2019. "Generalizing from Survey Experiments Conducted on Mechanical Turk: A Replication Approach." Political Science Research and Methods 7(3):613-628.

Coppock, Alexander and Oliver A McClellan. 2019. "Validating the demographic, political, psychological, and experimental results obtained from a new source of online survey respondents." Research \& Politics 6(1):2053168018822174.

Coppock, Alexander, Thomas J. Leeper and Kevin J. Mullinix. 2018. "Generalizability of heterogeneous treatment effect estimates across samples." Proceedings of the National Academy of Sciences 115(49):12441-12446. 
Cronbach, Lee J. and Karen. Shapiro. 1982. Designing evaluations of educational and social programs. San Francisco: Jossey-Bass.

Findley, Michael G, Kyosuke Kikuta and Michael Denly. 2020. "External Validity." Annual Review of Political Science .

Gadarian, Shana Kushner and Bethany Albertson. 2014. "Anxiety, Immigration, and the Search for Information." Political Psychology 35(2):133-164.

Glass, Gene V. 1976. "Primary, secondary, and meta-analysis of research." Educational researcher 5(10):3-8.

Huber, Gregory A and Celia Paris. 2013. "Assessing the programmatic equivalence assumption in question wording experiments: Understanding why Americans like assistance to the poor more than welfare." Public Opinion Quarterly 77(1):385-397.

IJzerman, Hans, Neil A Lewis, Andrew K Przybylski, Netta Weinstein, Lisa DeBruine, Stuart J Ritchie, Simine Vazire, Patrick S Forscher, Richard D Morey, James D Ivory et al. 2020. "Use caution when applying behavioural science to policy." Nature Human Behaviour 4(11):1092-1094.

Kane, John V., Yamil R. Velez and Jason Barabas. 2020. "Analyze the Attentive and Bypass Bias: Mock Vignette Checks in Survey Experiments." Unpublished Manuscript .

Klein, Richard A, Kate A Ratliff, Michelangelo Vianello, Reginald B Adams Jr, Štěpán Bahník, Michael J Bernstein, Konrad Bocian, Mark J Brandt, Beach Brooks, Claudia Chloe Brumbaugh et al. 2014. "Investigating variation in replicability." Social psychology .

Klein, Richard A, Michelangelo Vianello, Fred Hasselman, Byron G Adams, Reginald B Adams Jr, Sinan Alper, Mark Aveyard, Jordan R Axt, Mayowa T Babalola, Štěpán Bahník et al. 2018. "Many Labs 2: Investigating variation in replicability across samples and settings." Advances in Methods and Practices in Psychological Science 1(4):443-490.

Montgomery, Jacob M, Brendan Nyhan and Michelle Torres. 2018. "How conditioning on posttreatment variables can ruin your experiment and what to do about it." American Journal of Political Science 62(3):760-775. 
Mullinix, Kevin J., Thomas J. Leeper, James N. Druckman and Jeremy Freese. 2015. "The Generalizability of Survey Experiments." Journal of Experimental Political Science 2:109138.

Munger, Kevin. 2020. "Knowledge Decays: Temporal Validity and Social Science in a Changing World." Unpublished Manuscript .

Open Science Collaboration. 2015. "Estimating the reproducibility of psychological science." Science 349(6251).

Oppenheimer, Daniel M, Tom Meyvis and Nicolas Davidenko. 2009. "Instructional manipulation checks: Detecting satisficing to increase statistical power." Journal of experimental social psychology 45(4):867-872.

Paolacci, Gabriele, Jesse Chandler, Panagiotis G Ipeirotis et al. 2010. "Running experiments on Amazon Mechanical Turk." Judgment and Decision Making 5(5):411-419.

Permut, Stephanie, Matthew Fisher and Daniel M Oppenheimer. 2019. "Taskmaster: A tool for determining when subjects are on task." Advances in Methods and Practices in Psychological Science 2(2):188-196.

Peyton, Kyle. 2020. "Does Trust in Government Increase Support for Redistribution? Evidence from Randomized Survey Experiments." American Political Science Review 114(2):596-602.

Rosenfeld, Daniel L, Emily Balcetis, Brock Bastian, Elliot Berkman, Jennifer Bosson, Tiffany Brannon, Anthony L Burrow, Daryl Cameron, CHEN Serena, Jonathan E Cook et al. forthcoming. "Conducting Social Psychological Research in the Wake of COVID-19." Perspectives on Psychological Science. .

Tversky, Amos and Daniel Kahneman. 1981. "The framing of decisions and the psychology of choice." science 211(4481):453-458.

Valentino, Nicholas A., Antoine J. Banks, Vincent L. Hutchings and Anne K. Davis. 2009. "Selective Exposure in the Internet Age: The Interaction between Anxiety and Information Utility." Political Psychology 30(4):591-613.

Young, Lauren E. 2019. "The Psychology of State Repression: Fear and Dissent Decisions in Zimbabwe." American Political Science Review 113(1):140-155. 


\section{Online Appendix for: \\ The Generalizability of Online Experiments Conducted During The COVID-19 Pandemic}

\section{Contents}

A Individual Studies $\quad 1$

A.1 Russian reporters and American news . . . . . . . . . . . . . . . . . 1

A.2 Effect of framing on decision making . . . . . . . . . . . . . . . . . 2

A.3 Gain versus loss framing . . . . . . . . . . . . . . . . . . . . . . . 4

A.4 Welfare versus aid to the poor .................. 6

A.5 Gain versus loss framing with party endorsements . . . . . . . . . . . . 10

A.6 Foreign aid misperceptions . . . . . . . . . . . . . . . . . . . . . 13

A.7 Perceived intentionality for side effects . . . . . . . . . . . . . . . . 14

A.8 Atomic aversion . . . . . . . . . . . . . . . . . . 17

A.9 Attitudes toward immigrants . . . . . . . . . . . . . . . . . . 21

A.10 Fake news corrections . . . . . . . . . . . . . . . . . . . . . . . . . . . . . . . . .

A.11 Inequality and System Justification . . . . . . . . . . . . . . . . . . . . 26

A.12 Trust in government and redistribution . . . . . . . . . . . . . . . . 28

$\begin{array}{ll}\text { B Covariate distributions } & 31\end{array}$

C Treatment descriptions $\quad 38$

C.1 Attention Check Questions . . . . . . . . . . . . . . . 43

\section{List of Figures}

A.1 Effect of question ordering on support for Russian journalists in U.S. . . . . 2

A.2 Effect of "Cheap" vs. "Expensive" frame on decision to travel . . . . . . . . 4

A.3 Effect of gain vs. loss frame in "Asian disease" problem . . . . . . . . . . . . 6 
A.4 Effect of "Aid to Poor" vs. "Welfare" on support for government spending . 9

A.5 Effect of gain vs. loss framing experiment with party endorsement . . . . . . 12

A.6 Effect of policy-specific information on support for foreign aid . . . . . . . . 14

A.7 Effect of Harm vs. Help frame on perceived intentionality . . . . . . . . . . . 16

A.8 Support for prospective U.S. strike on Al Queda nuclear weapons lab in Syria 20

A.9 Support for retrospective U.S. strike on Al Queda nuclear weapons lab in Syria 21

A.10 Effects of immigrant attributes on support for admission to U.S. . . . . . . . 24

A.11 Effect of corrections on agreement with inaccurate statements . . . . . . . 26

A.12 Effect of "high inequality" treatment on comprehension questions and system justification scales . . . . . . . . . . . . . . . . . . . . 28

A.13 Effect of corruption information on trust in government and support for re-

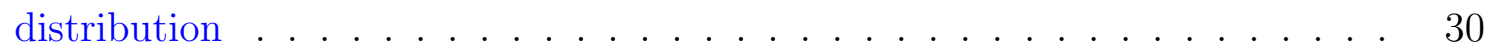

B.1 Region proportions by sample . . . . . . . . . . . . . . . . . 31

B.2 Education proportions by sample . . . . . . . . . . . . . . . 32

B.3 Household income proportions by sample . . . . . . . . . . . . . 33

B.4 Age proportions by sample . . . . . . . . . . . . . . . . 34

B.5 Male v. Female proportions by sample . . . . . . . . . . . . . . . 35

B.6 Race/Ethnicity proportions by sample . . . . . . . . . . . . . . . . 35

B.7 Partisanship proportions by sample . . . . . . . . . . . . . . . . 36

B.8 Voting behavior in 2016 proportions by sample . . . . . . . . . . . . . 37

C.1 Effect of framing on decision making: cheap condition (original) . . . . . . . 38

C.2 Effect of framing on decision making: expensive condition (original) . . . . . 38

C.3 Effect of framing on decision making: cheap condition (modified) . . . . . . . 38

C.4 Effect of framing on decision making: expensive condition (modified) . . . . 38

C.5 Perceived intentionality for side effects: helped condition (original) . . . . . . 39

C.6 Perceived intentionality for side effects: harmed condition (original) . . . . . 40

C.7 Perceived intentionality for side effects: helped condition (modified) . . . . . 41

C.8 Perceived intentionality for side effects: harmed condition (modified) . . . . 42

C.9 Pre-ACQ article for "Easy" and "Medium" ACQ . . . . . . . . . . . . . 43

C.10 "Easy" and "Medium" ACQ with correct responses highlighted . . . . . . . 44

C.11 "Hard" ACQ with correct response highlighted . . . . . . . . . . . . . . . . . 44 


\section{A Individual Studies}

Here we provide a detailed description of each study, their pre-COVID estimates, and the individual replication estimates that we obtained. All within-study estimates for non-binary outcomes are standardized using Glass's $\Delta$, which scales outcomes by the standard deviation in the control group (Glass, 1976). For all binary outcomes (e.g., the conjoint experiment) we report unstandardized estimates as these have a straightforward interpretation as the percentage point difference between the reference category and the level(s) of treatment.

\section{A.1 Russian reporters and American news}

This classic study by Hyman and Sheatsley (1950) shows that American subjects express more tolerance for Russian journalists to "come in here and send back to their papers the news as they see it" if they are first asked whether American journalists should be allowed to operate in Russia. The operating principle seems to be one of reciprocal fairness - after affirming that American journalists should be allowed to work in Russia, subjects appear to feel constrained to allow Russian journalists to work in America.

Pooling across the pre-COVID studies, the summary effect size estimate is a 30 percentage point increase in support for Russian journalists. Our effect estimate of 25.5 points is $83 \%$ of this magnitude, and this difference of 5.5 percentage points is not statistically significant $(P=0.16)$. Our replication estimate is also not statistically distinguishable from the two earlier replications reported in Schuman and Presser (1996). The baseline levels of support for Russian journalists in the control condition among Americans in 1950 (36\%), 1980 (55\%) and $1983(44 \%)$ are quite similar to COVID-era Lucid respondents (45\%). 
Figure A.1: Effect of question ordering on support for Russian journalists in U.S.

$\begin{array}{rc}\text { Hyman and Sheatsley (1950) } & 1 \\ \text { Schuman and Presser (1980) } & 1 \\ \text { Schuman et al. (1983) } & 1 \\ & \\ \text { Week } 3 & \\ & 0 \%\end{array}$

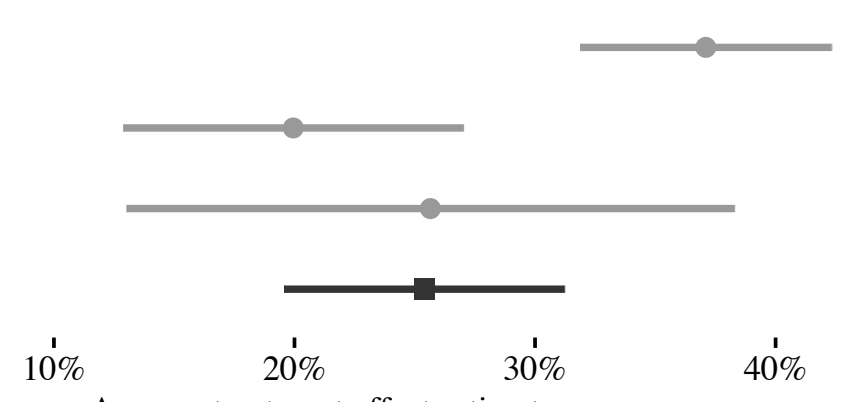

Average treatment effect estimate

\section{A.2 Effect of framing on decision making}

In a classic framing experiment by Tversky and Kahneman (1981, Study 10), undergraduates were instructed to imagine a scenario in which they were buying two items, one for $\$ 15$ and another for $\$ 125$. Participants in the "cheap" condition read the following prompt, with those in the "expensive" condition seeing the prices in parentheses: "Imagine that you are about to purchase a jacket for $\$ 125(\$ 15)$, and a calculator for $\$ 15(\$ 125)$. The salesman informs you that the calculator you wish to buy is on sale for $\$ 10(\$ 120)$ at the other branch of the store, located 20 minutes drive away. Would you make the trip to the other store?"

Although the total cost of both items was $\$ 140$ in each condition, with a potential of $\$ 5$ in savings for traveling, $68 \%$ of participants said they would travel when they could save $\$ 5$ on the $\$ 15$ item, whereas $29 \%$ said they would travel when they could save $\$ 5$ on the $\$ 125$ item. According to Tversky and Kahneman (1981), this difference of 39 percentage points illustrates how individuals' assess the potential gains and losses of outcomes in relative, rather than absolute, terms. When paying $\$ 15$ for an item, a $\$ 5$ discount seems substantial, whereas a $\$ 5$ discount on a $\$ 125$ item seems negligible.

This experiment has been replicated numerous times in both student and online samples. We use a slightly modified version of the original study from Klein et al. (2018) as our preCOVID benchmark for online samples. In this study, participants were presented with the 
following prompt, with those in the "expensive" condition seeing the prices in parentheses: "Imagine that you are about to purchase a ceramic vase for $\$ 250$ (\$30), and a wall hanging for $\$ 30$ ( $\$ 250)$. The salesman informs you that the wall hanging you wish to buy is on sale for $\$ 20(\$ 240)$ at the other branch of the store, located 20 minutes drive away. Would you make the trip to the other store?"

In the replication by Klein et al. (2018), 49\% of participants said they would travel to save $\$ 10$ on the "cheap" wall-hanging whereas 32\% said they would travel to save $\$ 10$ on the "expensive" wall-hanging. In our Week 7 replication study, half the participants were assigned to an experiment using this same scenario (wall hanging and ceramic vase). The other half were assigned to a COVID-specific scenario, where "ceramic vase" was replaced with "a box of Clorox disinfecting wipes," and "wall hanging" was replaced with "a box of N-95 respirator masks". See Figures C.1-C.4 for a full description of each condition.

Figure A.2 plots estimated treatment effects from the original study (student sample) alongside the pre-COVID replication (online sample) and our replication. The estimated effect in our direct replication of 15 percentage points was indistinguishable from the preCOVID replication (16 percentage points). For the COVID-specific experiment, the estimated treatment effect of 7 percentage points was indistinguishable from zero, and smaller than both the pre-COVID replication (difference of 9 percentage points, $\mathrm{SE}=0.05, P=0.02$ ) and our direct replication (difference of 8 percentage points, $\mathrm{SE}=0.06, P=0.09$ ). Pooling across the pre-COVID replication and original, the summary effect size estimate is 0.17 $(\mathrm{SE}=0.01, P<0.01)$, compared to $0.11(\mathrm{SE}=0.03, P<0.01)$ for our replications for a correspondence of $64 \%$. 
Figure A.2: Effect of "Cheap" vs. "Expensive" frame on decision to travel

Tversky and Kahneman (1981)

Many Labs (2018)

Direct replication

COVID-specific

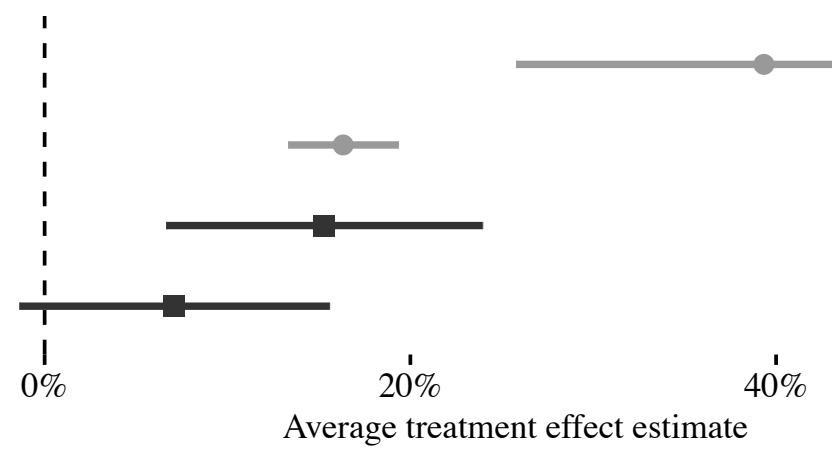

Although our replications estimates are, on average, smaller than the pre-COVID average, the direct replication closely approximates the 2018 study and all replication estimates are in the expected direction. We also note that the estimated effect from the COVID-specific replication is smaller but statistically indistinguishable from the direct replication. This raises the possibility that the COVID-specific language in the replication slightly decreased the power of the framing effect. We nevertheless conclude that the replications were successful.

\section{A.3 Gain versus loss framing}

In this classic framing experiment by Tversky and Kahneman (1981, Study 1), undergraduates were instructed to imagine the U.S. was preparing for the outbreak of an unusual "Asian disease", which was expected to kill 600 people. In the "gain" framing condition,

participants were asked to select between two courses of action to combat the disease: if Program A is adopted, 200 people will be saved; if Program B is adopted, there is a 1/3 probability that 600 people will be saved, and $2 / 3$ probability that no people will be saved. In the "loss" framing condition, participants were asked to select between two different formulations: if Program A is adopted, 400 people will die; if Program B is adopted, there is a $1 / 3$ probability that nobody will die, and $2 / 3$ probability that 600 people will die.

In both framing conditions, the expected number of deaths is 400 for both Program A 
and Program B. In the original study, $72 \%$ selected Program A in the gain frame, whereas $22 \%$ selected Program A in the loss frame, for an estimated treatment effect of 50 percentage points. According to Tversky and Kahneman (1981), the observed preference reversal illustrates how individuals' choices involving gains are risk averse whereas choices involving losses are risk seeking.

This experiment has been widely replicated across time in both student samples and online samples. Figure A.3 plots estimated treatment effects from the original study (student sample) alongside those obtained from pre-COVID studies (online samples) in the Many Labs replication project (Klein et al., 2014) in 2013, in 2013 on MTurk (Berinsky, Huber and Lenz, 2012), in 2016 on Lucid (Coppock and McClellan, 2019), and in five of our replications. The first four of our replications are COVID-specific versions of the original. Participants were instead asked to imagine that "the Mayor of a U.S. city is preparing for another outbreak of the novel coronavirus in the Spring of 2021, which is expected to kill 600 city residents." The fifth replication is a direct replication of the pre-COVID experiments using the original wording. 
Figure A.3: Effect of gain vs. loss frame in "Asian disease" problem

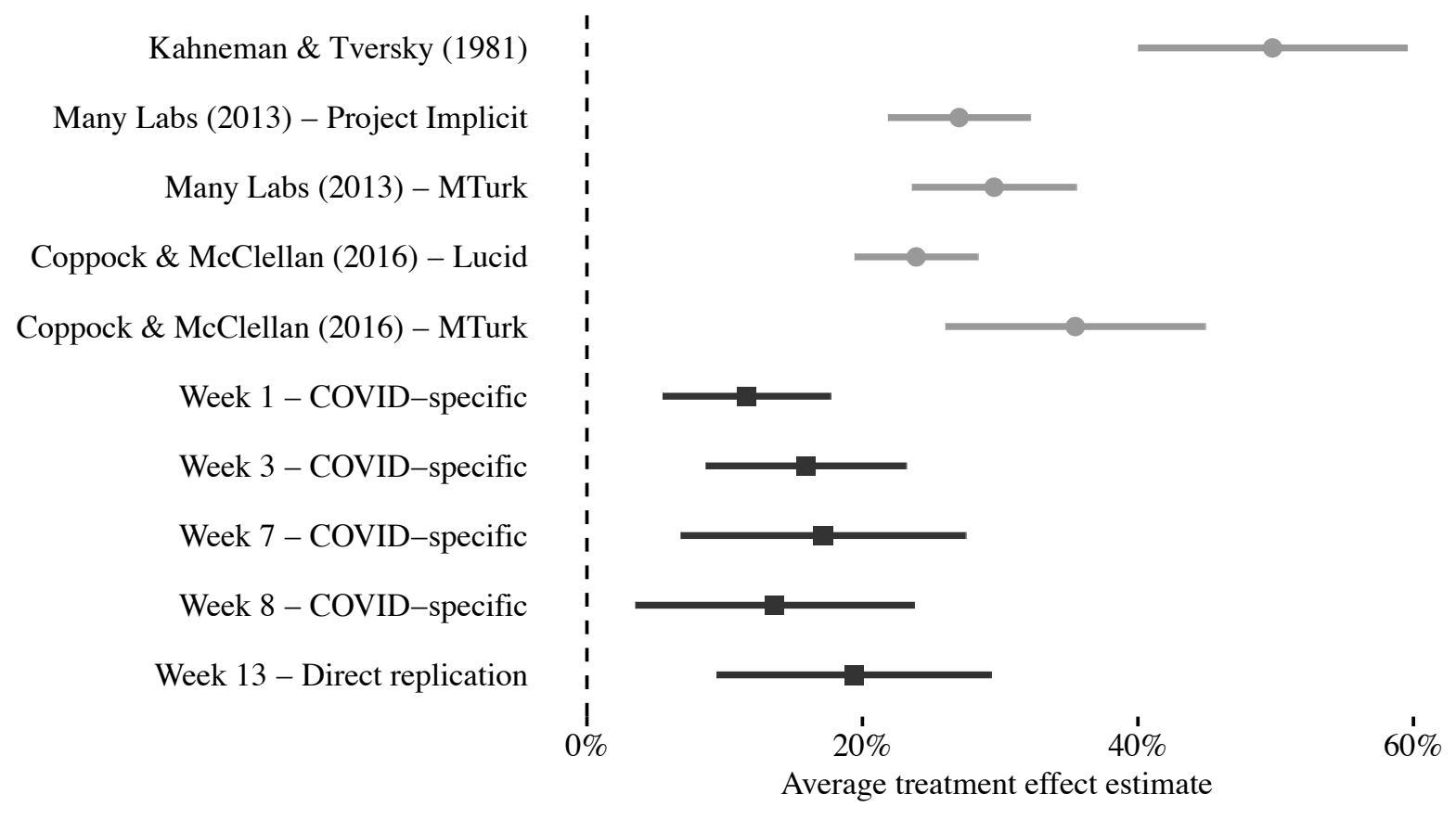

The summary effect size for our five replications is $0.15(\mathrm{SE}=0.02, P<0.01)$, approximately $50 \%$ the size of the summary effect size for the five pre-COVID experiments (summary effect size: $0.29, \mathrm{SE}=0.01, P<0.01$ ). Although the replications estimates are, on average, smaller than those from pre-COVID experiments (difference: 0.14, SE $=0.02$, $P<0.01$, all COVID-era replication estimates are statistically distinguishable from zero, and in the expected direction. We therefore conclude that the replications were successful, regardless of whether COVID-specific language was used in the scenario description.

\section{A.4 Welfare versus aid to the poor}

The large effect of describing government assistance as "welfare" rather than "aid to the poor" is one of the most robust experimental findings in political science. In the original experiment (Smith, 1987), a sample of U.S. adults from the General Social Survey (GSS) were asked by telephone survey whether they believed there was "too much", "about the 
right amount", or "too little" spending across multiple issues, with social welfare benefits being described as "assistance for the poor" in the treatment arm and "welfare" in the control arm. This experiment has been replicated on GSS surveys from 1986 to 2018. Respondents are consistently more supportive of government spending on "assistance for the poor" than "welfare". 1

Figure A.4 plots estimated treatment effects from nine replications of the original experiment on GSS respondents using computer-assisted personal interviews (CAPI) and paper and pencil interviews (PAPI), alongside those obtained from our twelve replications, and two pre-COVID replications, using computer-assisted web interviews (CAWI). Following prior replications on online samples (e.g., Huber and Paris, 2013), responses are coded as -1 ("too much"), 0 ("about the right amount"), and 1 ("too little"). Estimated treatment effects are standardized using Glass's $\Delta$, and positive estimates indicate respondents are more supportive of spending on "Aid to the Poor" than "Welfare". Two of our replications (Week 9 and Week 5) were within-subject experiments that asked respondents both spending questions in randomized order. All replication estimates are statistically distinguishable from zero and are in the expected direction. ${ }^{2}$

The summary effect size for the experimental estimates from CAWI surveys is 0.44 (SE $=0.02, P<0.01$ ), approximately $44 \%$ the size of the summary effect sizes for the CAPI (1.00, SE $=0.01, P<0.01)$ and PAPI $(1.05, \mathrm{SE}=0.01, P<0.01)$ surveys from the GSS. Within the CAWI surveys, however, our experimental estimates (summary effect size: 0.43 , $\mathrm{SE}=0.02, P<0.01)$ are indistinguishable from the pre-COVID estimates $(0.46, \mathrm{SE}=0.05$, $P<0.01)$ from Huber and Paris (2013). Unlike in the CAWI surveys, there is also strong

\footnotetext{
${ }^{1}$ See Huber and Paris (2013) for evidence that individuals believe these labels describe different social programs.

${ }^{2}$ Interestingly, the non-experimental within-subjects estimates are solidly in line with the experimental estimates, suggesting that subjects feel no pressure to keep their support for welfare consistent with their support for aid to the poor. This pattern contrasts strongly with the evident pressure for consistency in the Russian journalists experiment. For further discussion on tradeoffs in the choice of within versus between subjects designs, see Clifford, Sheagley and Piston (2020).
} 
evidence of statistical heterogeneity among estimates from the CAPI and PAPI surveys. ${ }^{3}$

Given the variation in GSS question wordings, survey modes, and estimates across time, we benchmark against the original 1986 estimate and the two replications on online samples from Huber and Paris (2013). The summary effect size for these three estimates is 0.70 (SE $=0.04, P<0.01$ ) and our COVID-era replication estimates are approximately $61 \%$ the size of this pre-COVID benchmark. We note that significant differences between estimates across CAWI, PAPI, and CAPI surveys was a feature of experimental research prior to COVID.

\footnotetext{
${ }^{3}$ CAWI $\chi^{2}: 12.72, P=0.31$, CAPI $\chi^{2}: 17.21, P=0.03$, PAPI $\chi^{2}: 85.19, P<0.01$
} 
Figure A.4: Effect of "Aid to Poor" vs. "Welfare" on support for government spending

\section{Computer-assisted web interviews}

Huber \& Paris (2013) - MTurk

Huber \& Paris (2013) - YouGov

Week 1

Week 2

Week 3

Week 4

Week 5

Week 6

Week 7

Week 8

Week 9

Week 11

Week 12

Week 13

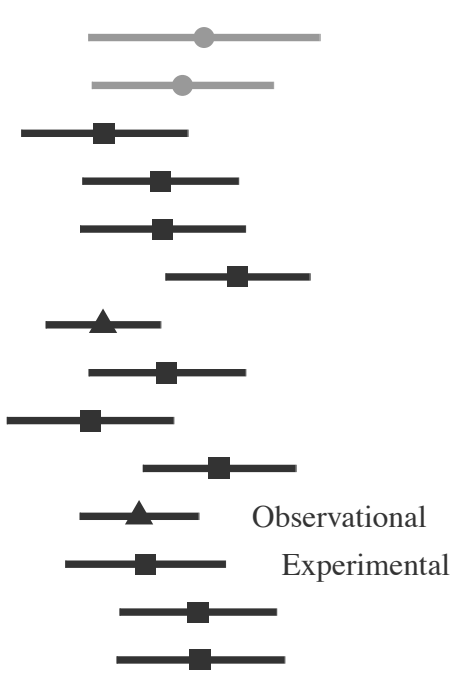

\section{Computer-assisted personal interviews}

GSS 2002
GSS 2004
GSS 2006
GSS 2008
GSS 2010
GSS 2012
GSS 2014
GSS 2016
GSS 2018

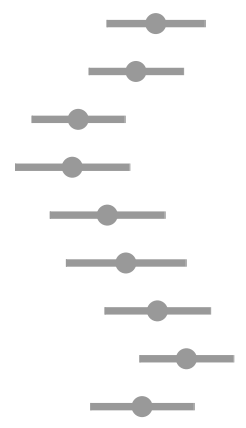

Paper and pencil interviews

GSS 1986
GSS 1987
GSS 1988
GSS 1989
GSS 1990
GSS 1991
GSS 1993
GSS 1994
GSS 1996
GSS 1998
GSS 2000

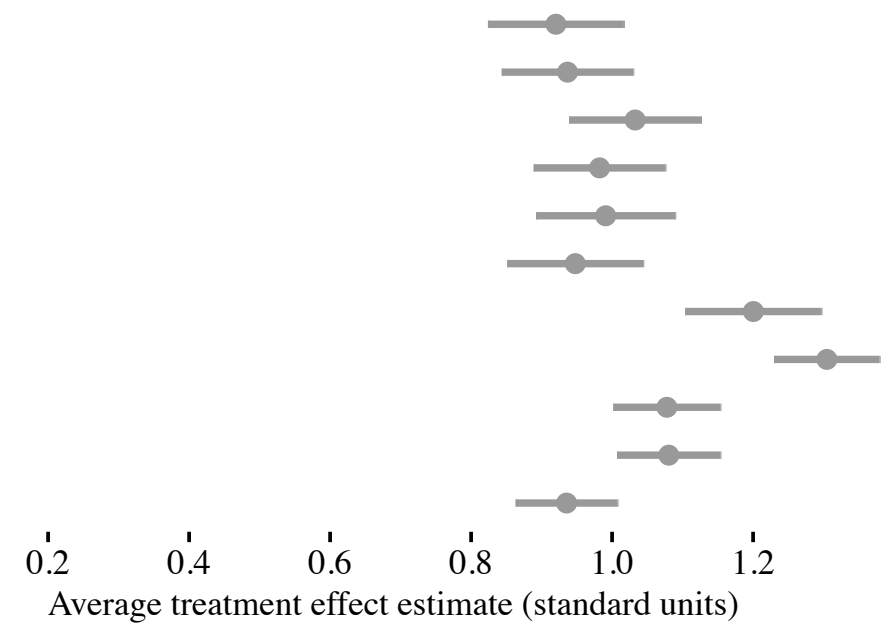

Notes: Starting in 2002, the GSS replaced "assistance to the poor" with "assistance for the poor." Week 13 is a direct replication of Huber and Paris (2013) using the GSS question wordings. The other replications use the ANES question wording, which asks whether respondents think spending should be "increased" (coded 1), "kept the same" (coded 0), or "decreased" (coded -1). 


\section{A.5 Gain versus loss framing with party endorsements}

Druckman (2001) extended the "Asian disease" protocol to explicitly incorporate political considerations. In his original study, a sample of undergraduates were randomly assigned to the classic version of the study or a modified version that randomly assigned party endorsements instead of "Program A" and "Program B". In the "gain" framing condition, participants were asked to select between two courses of action, with one of three randomly assigned labels: If [Program A, the Democrats' Program, the Republicans' Program] is adopted, 200

people will be saved; If [Program B, the Republicans' Program, the Democrats' Program], there is a $1 / 3$ probability that 600 people will be saved, and a $2 / 3$ probability that no people will be saved. In the "loss" framing condition, the descriptions were: If [Program A, the Democrats' Program, the Republicans' Program] is adopted, 400 people will die; If [Program B, the Republicans' Program, the Democrats' Program], there is a $1 / 3$ probability that nobody will die, and a $2 / 3$ probability that 600 people will die.

In the original study, the preference reversal effect from Tversky and Kahneman (1981) was replicated when "Program A" and "Program B" were used as labels. However, these effects were greatly attenuated (or indistinguishable from zero) when the programs were labeled with party endorsements. According to Druckman (2001), this difference illustrates how partisans' desire to choose their party's program can overwhelm preference reversals due to "pure" framing effects.

Figure A.5 plots estimated treatment effects from the original study (student sample) alongside three replications. Two of our replications (Week 7 and Week 8) are COVIDspecific versions of the original where "unusual Asian disease" was replaced with "another outbreak of the novel coronavirus". The Week 13 replication is a direct replication of the original Asian disease experiment. All estimates are statistically distinguishable from zero and in the expected direction when "Program A" is used to describe the "risk-averse alternative" (e.g. save 200 people versus 400 people will die). Consistent with the original 
experiment, however, adding the partisan labels attenuate (or eliminate) preferences reversals among partisans: among Democrats, preference reversal effects are indistinguishable from zero when "Program A" is replaced with "Republicans' Program"; among Republicans, preference reversal effects are indistinguishable from zero when "Program A" is replaced with "Democrats' Program".

Table A.5 provides a summary of differences between the original study and the replications by treatment arm. Although the replications estimates are, on average, smaller than those from the original study, all replication estimates are in the expected direction. We therefore conclude that the original study replicated. 
Figure A.5: Effect of gain vs. loss framing experiment with party endorsement

Framing effects by label of risk-averse alternative, among Democrats

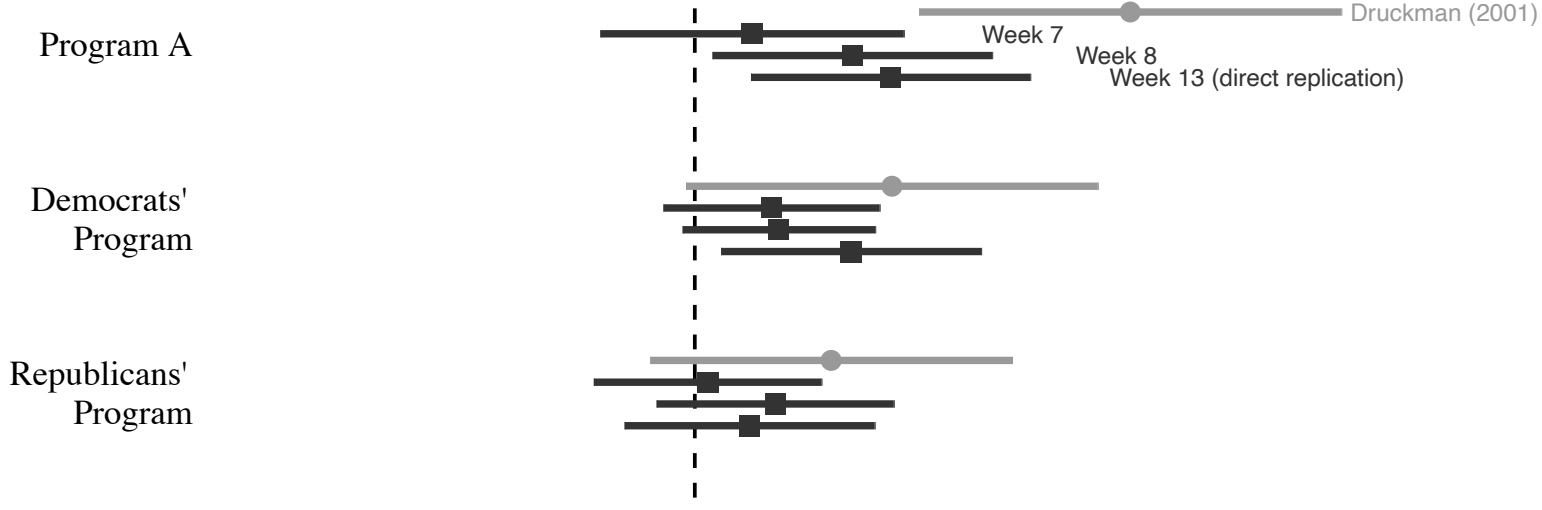

Framing effects by label of risk-averse alternative, among Republicans

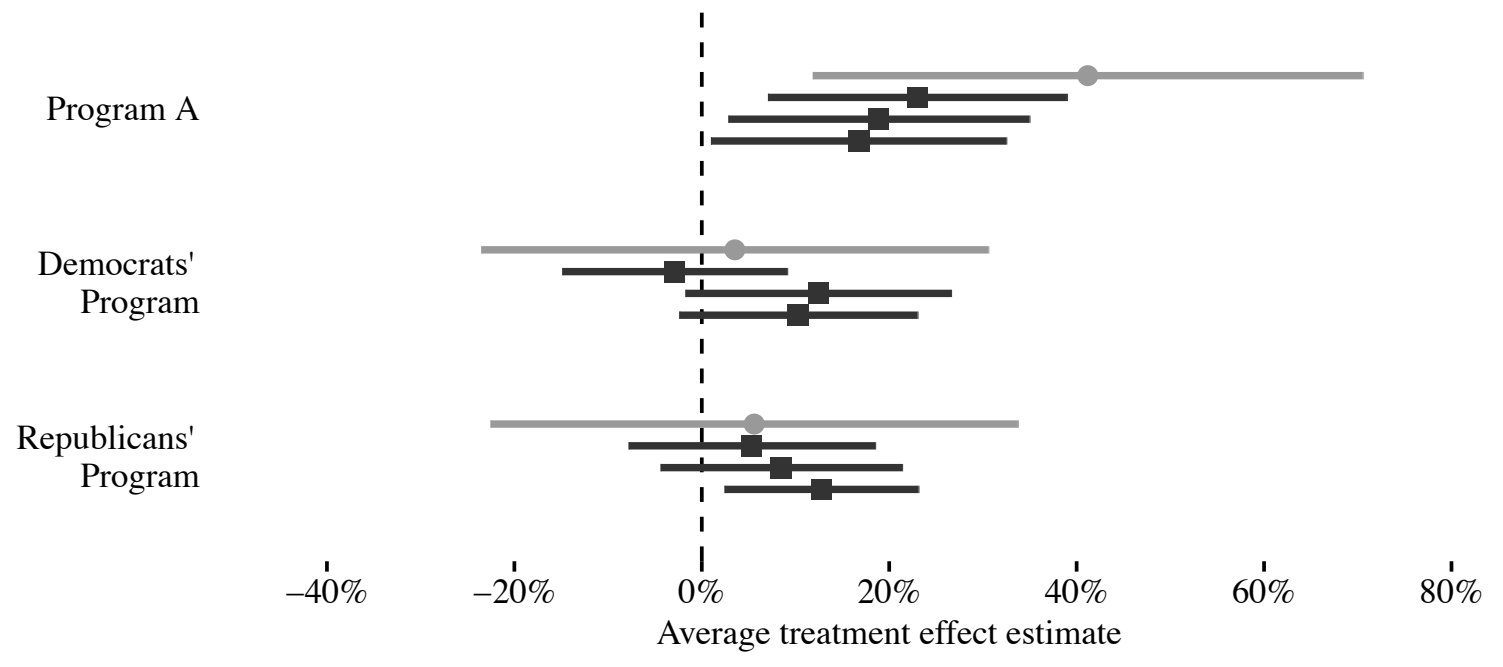


TABLE 1: Summary effect sizes in gain vs. loss framing experiment with party endorsement

\begin{tabular}{llllll}
\hline $\begin{array}{l}\text { Label of risk-averse } \\
\text { alternative }\end{array}$ & $\begin{array}{l}\text { Partisan } \\
\text { subgroup }\end{array}$ & $\begin{array}{l}\text { Replication } \\
\text { Summary }\end{array}$ & $\begin{array}{l}\text { Original } \\
\text { Study }\end{array}$ & Difference & $\begin{array}{l}\text { Relative } \\
\text { Effect Size }\end{array}$ \\
\hline Program A & Democrats & $0.15(0.04)^{*}$ & $0.46(0.11)^{*}$ & $-0.31(0.12)^{*}$ & 0.32 \\
Program A & Republicans & $0.20(0.05)^{*}$ & $0.41(0.15)^{*}$ & $-0.22(0.15)$ & 0.47 \\
Democrats' Program & Democrats & $0.11(0.03)^{*}$ & $0.21(0.11)$ & $-0.11(0.12)$ & 0.50 \\
Democrats' Program & Republicans & $0.06(0.04)$ & $0.04(0.13)$ & $0.02(0.14)$ & 1.66 \\
Republicans' Program & Democrats & $0.05(0.04)$ & $0.15(0.10)$ & $-0.09(0.10)$ & 0.35 \\
Republicans' Program & Republicans & $0.10(0.03)^{*}$ & $0.06(0.14)$ & $0.04(0.14)$ & 1.70 \\
\hline
\end{tabular}

Notes: Relative size is the summary effect size divided by the original effect size: values less than 1 indicate summary effect sizes smaller than original effect sizes. $P<0.05^{*}$.

\section{A.6 Foreign aid misperceptions}

In the original experiment (Gilens, 2001), respondents from a nationally representative telephone survey fielded in 1998 were queried about their support for spending on foreign aid after being read a randomly assigned prompt about a hypothetical news story. In the control condition, the prompt read "The story is about a news report that was just released about American foreign aid to help other countries. Have you heard about this story?" In the treatment condition, the prompt added factual information designed to correct misperceptions about foreign aid spending: "It said that the amount of money we spend for foreign aid has been going down and now makes up less than one cent of every dollar that the federal government spends."

Following the prompt, respondents were asked: "How do you feel about the amount of money the federal government (in Washington) spends on foreign aid to other countries? Do you think the federal government should spend more of foreign aid, less, or about the same as it does not?" The original study reported that respondents in the treatment condition 
were 16.6 percentage points less likely to support cuts for foreign aid than respondents in the control group. According to Gilens (2001), this illustrates that the general public over-estimates the percentage of the budget allocated to foreign aid, but correcting this misperception with policy-specific information can decrease opposition to foreign aid.

In the original study, support for foreign aid was measured on a 3-point scale: "Less" (coded -1), "About the same" (coded 0), "More" (coded 1). The 16.6 percentage point effect reported in Gilens (2001) was obtained from a logistic regression of the binary treatment indicator on a truncated outcome, i.e. $Y_{i}=1$ if respondent selected "Less"; 0otherwise, and a variety of control variables. In our reanalysis of the original data, we estimate treatment effects using difference-in-means with the raw three-point outcome variable.

Figure A.6 plots estimated treatment effects from the original study (telephone interview) alongside our replication (online interview). The estimated treatment effect in the original study is an increase in support for foreign aid of 0.21 ( $\mathrm{SE}=0.06, P=<0.01$ ). The estimated treatment effect in the replication study is a decrease in support for foreign aid by 0.05 (SE $=0.06, P=0.40)$. The estimate from the original study is therefore 0.26 units larger - in the opposite direction - than the replication study $(\mathrm{SE}=0.09, P<0.01$ ). This is the only experimental result among our set that we classify as a replication failure.

Figure A.6: Effect of policy-specific information on support for foreign aid

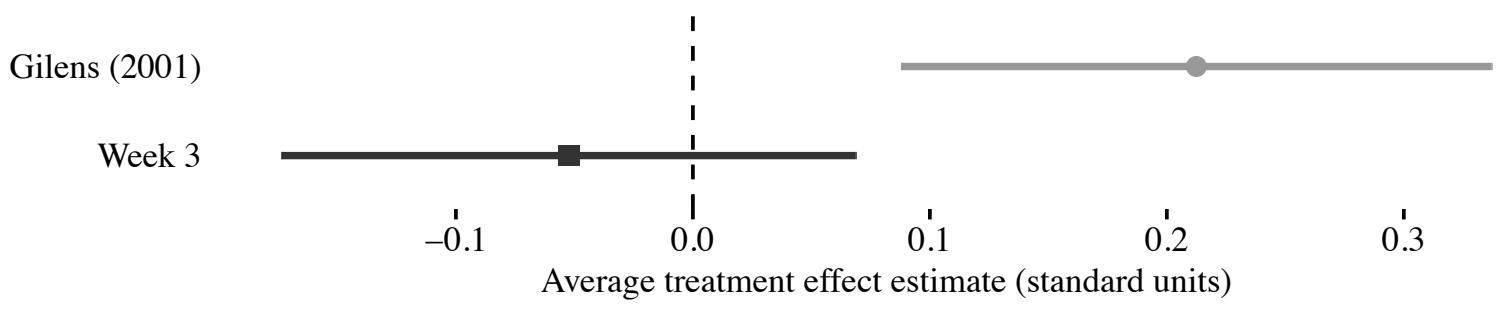

\section{A.7 Perceived intentionality for side effects}

In the original study (Knobe, 2003, Study 1), individuals were recruited from a New York City park to participate in an experiment based on the following vignette: 
The vice-president of a company went to the chairman of the board and said, "We are thinking of starting a new program. It will help us increase profits, and it will also help the environment."

The chairman of the board answered, "I don't care at all about helping the environment. I just want to make as much profit as I can. Let's start the new program."

Respondents assigned to the Help condition read that the chairman's decision had a helpful side effect: "They started the new program. Sure enough, the environment was helped." Those assigned to the Harm condition read "They started the new program. Sure enough, the environment was harmed." In the Help condition, $23 \%$ of subjects agreed with the statement "The chairman helped the environment intentionally," whereas $82 \%$ of those in the Harm condition agreed that "The chairman harmed the environment intentionally." According to Knobe (2003), this estimated treatment effect of 59 percentage points illustrates how the perceived intentionality of individual actions depends upon whether their consequences are helpful or harmful.

This experiment has been replicated multiple times in both student and online samples. We use the replication study from Klein et al. (2018) - which found an estimated treatment effect of 64 percentage points - as our pre-COVID benchmark for online samples. In our replications, half the participants were assigned to an experiment using this same scenario as the original study. The other half were assigned to a COVID-specific scenario, based on the following vignette:

The vice-president of a company went to the chairman of the board and said, "We are thinking of marketing a new drug to treat COVID-19. It will help us increase profits, and the drug will also help older people with heart conditions."

The chairman of the board answered, "I don't care at all about helping older people with heart conditions. I just want to make as much profit as I can. Let's start marketing the new drug." 
Those assigned to the Help condition read that the chairman's decision had a harmful side effect: "They started marketing the new drug. Sure enough, older people with heart conditions were helped." Those assigned to the Harm condition instead read that older people with heart conditions were "harmed". See Figures C.5-C.8 for full text of each condition.

Figure A.7 plots estimated treatment effects from the original study (sample from Manhattan park) alongside the Many Labs (2018) replication (online sample) and our replications (online sample). The estimated treatment effect is $0.39(\mathrm{SE}=0.04, P<0.01)$ in the direct replication and 0.38 in the COVID-specific replication $(\mathrm{SE}=0.04, P<0.01)$. The estimated treatment effect for the COVID-specific replication is about 0.01 points smaller than the direct replication ( $\mathrm{SE}=0.04, P=0.46$ ). The direct replication is approximately $60 \%$ the size of the pre-COVID benchmark (difference of 0.25 points, $\mathrm{SE}=0.04, P<0.01$ ). The replication estimates are considerably smaller than the pre-COVID benchmark, but all estimates are in the expected direction and statistically distinguishable from zero. We therefore conclude that the pre-COVID study replicated, regardless of whether COVID-specific language was used in the vignettes.

Figure A.7: Effect of Harm vs. Help frame on perceived intentionality

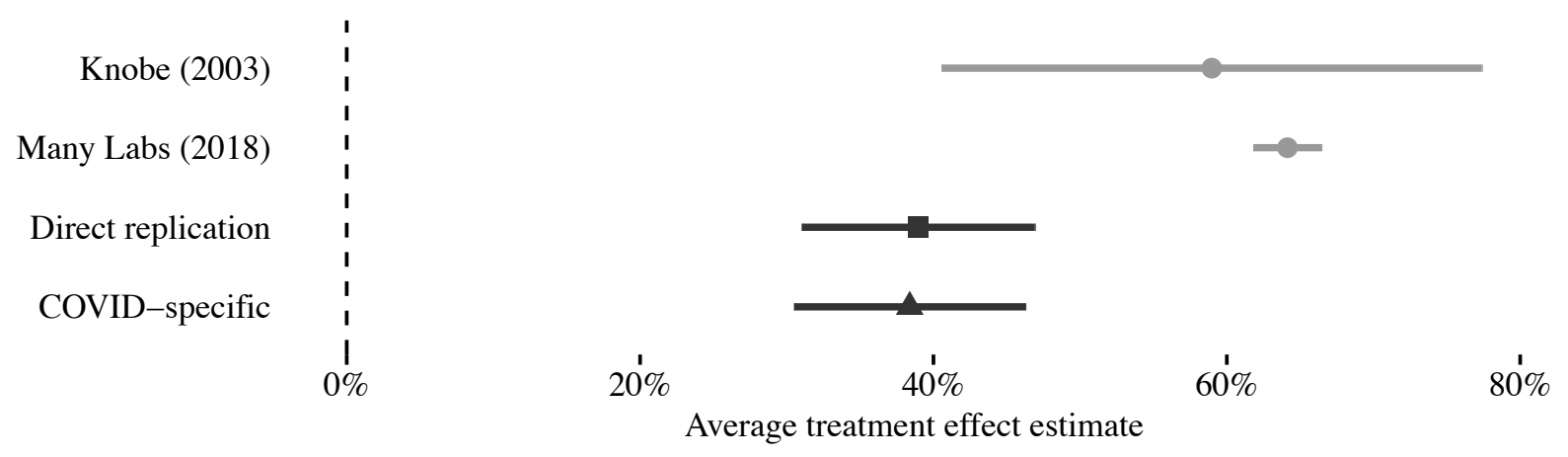




\section{A.8 Atomic aversion}

In the original study (Press, Sagan and Valentino, 2013), a quota sample of online survey participants recruited by YouGov were randomly assigned to participate in one of two independent experiments. In the prospective experiment, subjects read a hypothetical news article that reported U.S. officials were deciding between nuclear and conventional military options for destroying an Al Qaeda nuclear weapons lab in Syria. The article compared the expected effectiveness of each military option, and estimated 1,000 Syrian civilian deaths regardless of which option was pursued. Within this experiment, subjects were assigned to one of three treatment arms that varied only in the likely success of the conventional strike: 1) a "90/90" condition in which the nuclear and conventional strike both had a $90 \%$ chance of success; 2) a " $90 / 70$ " condition in which the conventional strike had a $75 \%$ chance of success; 3) a " $90 / 45 "$ condition in which the conventional strike had a $45 \%$ chance of success. The relative effectiveness of each option was described in the article text, alongside a two-by-two matrix that compared the chances of success (90/90, 90/70, or 90/45) and estimated civilian causalities (fixed) for both options.

In the retrospective experiment, subjects read a hypothetical news article that described a U.S. military strike that had already been carried out on the Al Qaeda lab. Within this experiment, subjects were assigned to one of two treatment arms that described the weapons used to carry out the attack: 1) a "conventional strike" condition in which 100 conventional cruise missiles were used; 2) a "nuclear strike" condition in which 2 nuclear cruise missiles were used. The number of civilian casualties and the outcome (the lab was successfully destroyed) were fixed across conditions.

In both experiments, all subjects were informed prior to random assignment that, if they failed to pass comprehension questions about the article, they could be ineligible to finish. If they instead answered the comprehension questions correctly, they were told they would

be eligible to participate in a raffle for a $\$ 100$ gift certificate. subjects who failed to pass the 
post-treatment comprehension questions were excluded from the analysis sample.

Aronow, Baron and Pinson (2019) noted this practice of dropping subjects who fail posttreatment "manipulation checks" can induce bias in estimates of treatment effects. They replicated the original study on a sample of MTurk workers and found that retaining subjects who failed the comprehension questions resulted in different estimates than the original study. In this replication, subjects were also told in advance that they would be ineligible to complete the survey if they failed the comprehension questions, but were entered into a raffle for a $\$ 100$ bonus payment if they passed. In addition, respondents in the prospective experiment viewed a large version of the two-by-two graphic that appeared in the article after the comprehension questions were answered, but before viewing any outcome questions.

Lucid does not give researchers the ability to pay survey respondents bonuses, so no incentives could be offered for those who passed the comprehension questions in our replications. In addition, subjects in our replications of the prospective experiment viewed the article once, and a two-by-two graphic was not presented after the comprehension questions (as in Aronow, Baron and Pinson, 2019). All other design details were the same as in the original study.

Figure A.8 plots estimated treatment effects in the prospective experiment, with the 90/90 condition as the control group, for the original study, the replication by Aronow, Baron and Pinson (2019), and our three replications. In the original study, the 90/70 condition caused an increase in the proportion of subject that preferred the nuclear option by about 37 percentage points relative to the $90 / 90$ condition. The estimated effect of the $90 / 45$ condition, relative to the $90 / 90$ condition, was 51 percentage points. Similarly, the 90/70 condition caused an increase in the proportion of subjects that approved of the nuclear option by about 17 percentage points, and the $90 / 45$ condition caused an increase of about 27 percentage points. In other words, estimated treatment effects increased monotonically with the relative effectiveness of nuclear weapons. 
Estimated treatment effects in the pre-COVID replication by Aronow, Baron and Pinson (2019) were similar to the original study for both outcome measures. The estimated treatment effects in our replications were considerably smaller for the "Prefer Nuclear Use" outcome, but of the expected sign and statistically distinguishable from zero. Estimates for the "Approve Nuclear Use" outcome, however, were of the opposite sign and not distinguishable from zero in $2 / 3$ of our replications. Table 2 provides a summary of the differences in estimates from the prospective experiment in our replications, the original study, and the ABP replication.

Figure A.9 plots estimated treatment effects in the retrospective experiment, with the "conventional strike" condition as the control group. The original study reported that differences between the "nuclear strike" (treatment) and "conventional strike" (control) were "substantively small and not statistically significant" (Press, Sagan and Valentino, 2013, p. 197). The pre-COVID replication byAronow, Baron and Pinson (2019) found, however, that the nuclear strike caused a 12 percentage point reduction in the proportion of respondents who "approved" the strike, and a 13 percentage point reduction in the proportion who believed the strike was "ethical". Table 3 provides a summary of the differences in estimates from the retrospective experiment in our replications, the original study, and the ABP replication.

When compared to the original study and the ABP replication, our replication estimates for the "Prefer Nuclear Use" outcome in the prospective experiment are significantly smaller, but of the expected sign and statistically distinguishable from zero. The estimates for the "Approve Nuclear Use" outcome are, however, signed in the opposite direction and indistinguishable from zero. Estimates for both outcomes in the retrospective experiment are comparable to those reported in the original study and the ABP replication. We therefore conclude that the atomic aversion experiment was partially replicated. 
Figure A.8: Support for prospective U.S. strike on Al Queda nuclear weapons lab in Syria

Press et al. (2013)

Aronow et al. (2019)

Week 5

Prefer Nuclear Use

1
1
1

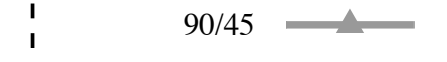

I

I

$90 / 70$

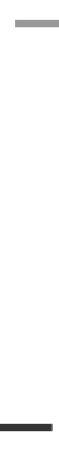

1

$+\frac{1}{1}$

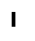

I

I

Week 6

Week 13

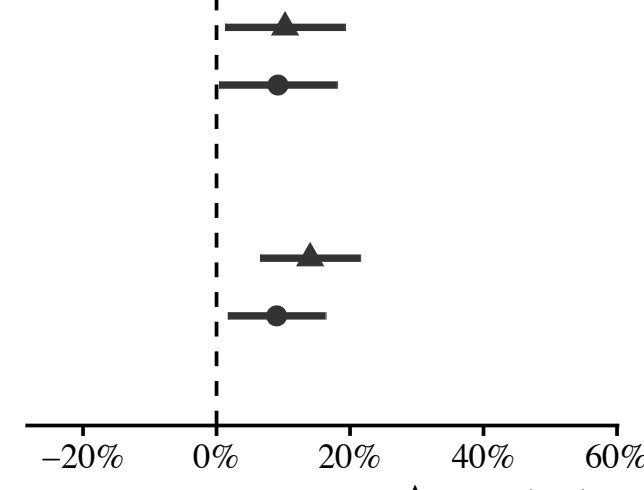

Average treatment effect
Approve Nuclear Use

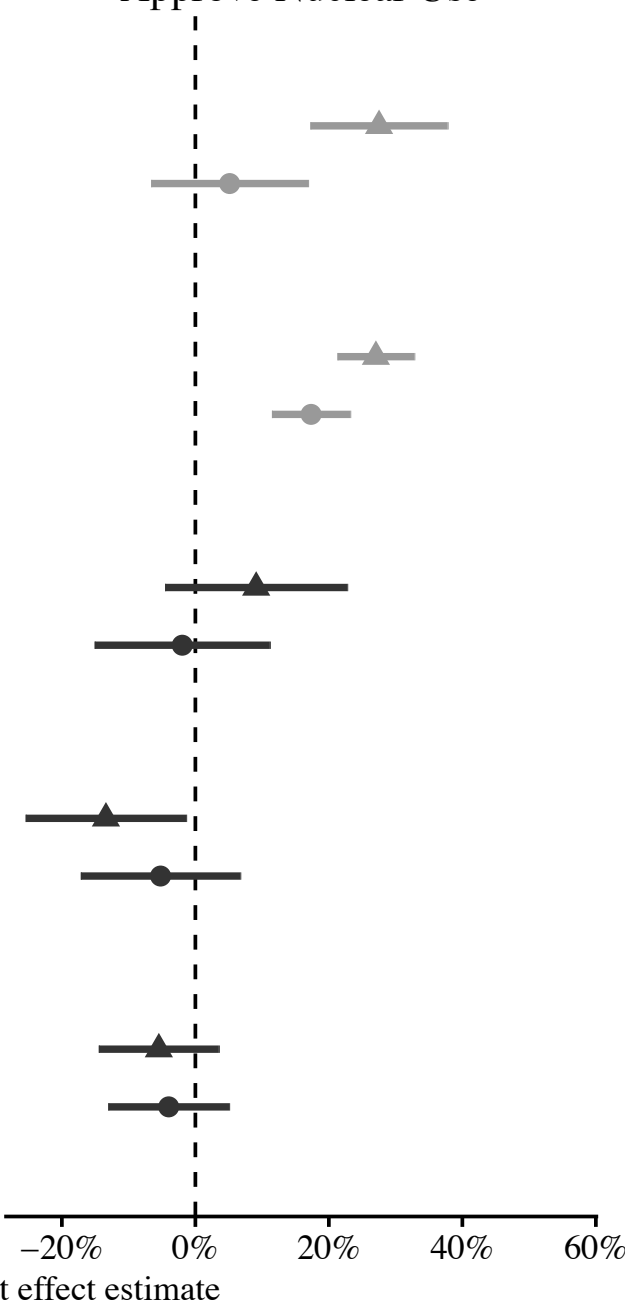

TABLE 2: Summary of estimates in prospective atomic aversion experiment

\begin{tabular}{llllllllll}
\hline Group & Outcome & $\begin{array}{l}\text { Replication } \\
\text { Summary }\end{array}$ & $\begin{array}{l}\text { Original } \\
\text { Study }\end{array}$ & Difference & $\begin{array}{l}\text { Relative } \\
\text { Size }\end{array}$ & $\begin{array}{l}\text { ABP } \\
\text { Replication }\end{array}$ & Difference & $\begin{array}{l}\text { Relative } \\
\text { Size }\end{array}$ \\
\hline $90 / 70$ & Prefer & $0.08(0.02)^{*}$ & $0.30(0.05)^{*}$ & $-0.22(0.06)^{*}$ & 0.27 & $0.37(0.03)^{*}$ & $-0.29(0.04)^{*}$ & 0.21 \\
$90 / 45$ & Prefer & $0.13(0.03)^{*}$ & $0.48(0.05)^{*}$ & $-0.35(0.06)^{*}$ & 0.27 & $0.51(0.03)^{*}$ & $-0.39(0.04)^{*}$ & 0.25 \\
\hline $90 / 70$ & Approve & $-0.04(0.03)$ & $0.05(0.06)$ & $-0.09(0.07)$ & - & $0.17(0.03)^{*}$ & $-0.21(0.04)^{*}$ & - \\
$90 / 45$ & Approve & $-0.05(0.03)$ & $0.28(0.05)^{*}$ & $-0.32(0.06)^{*}$ & - & $0.27(0.03)^{*}$ & $-0.32(0.04)^{*}$ & - \\
\hline
\end{tabular}

Notes: Relative effect sizes are the replication summary effect sizes divided by the ABP replication or original effect size: values less than 1 indicate summary effect size is smaller than the ABP or original effect size. Relative effect sizes are not calculated if replication estimates are the opposite sign of comparison estimates. $P<0.05^{*}$. 
Figure A.9: Support for retrospective U.S. strike on Al Queda nuclear weapons lab in Syria

Press et al. (2013)

Approve Strike

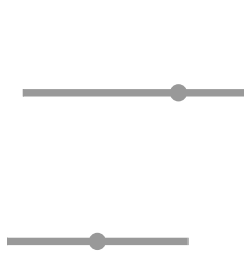

Aronow et al. (2019)

Week 5

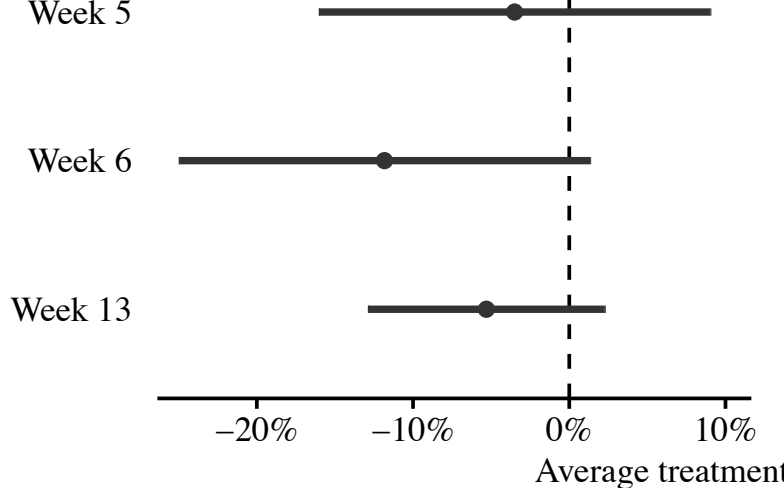

Ethical Strike

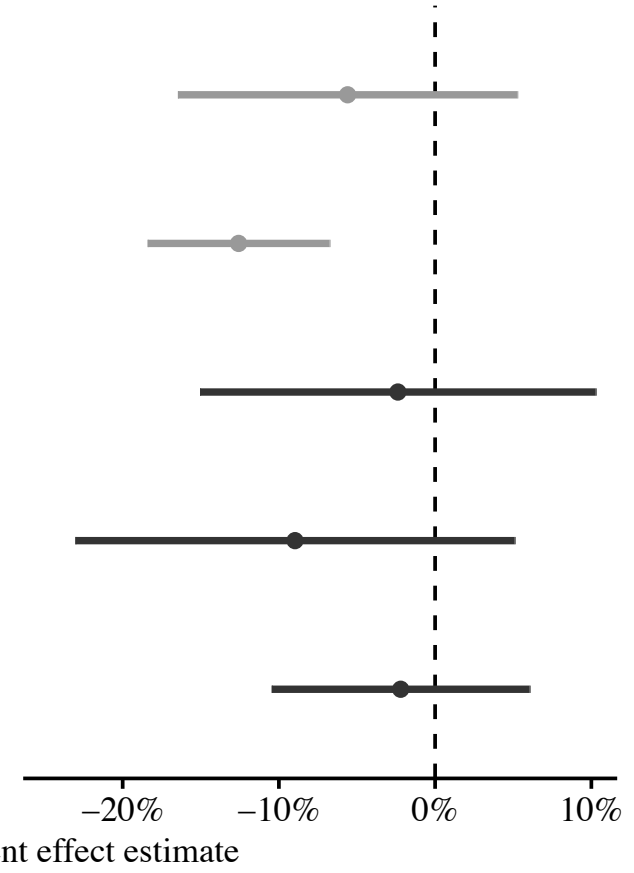

TABLE 3: Summary of estimates in retrospective atomic aversion experiment

\begin{tabular}{llllllll}
\hline Outcome & $\begin{array}{l}\text { Replication } \\
\text { Summary }\end{array}$ & $\begin{array}{l}\text { Original } \\
\text { Study }\end{array}$ & Difference & $\begin{array}{l}\text { Relative } \\
\text { Size }\end{array}$ & $\begin{array}{l}\text { ABP } \\
\text { Replication }\end{array}$ & Difference & $\begin{array}{l}\text { Relative } \\
\text { Size }\end{array}$ \\
\hline Approve & $-0.06(0.03)^{*}$ & $-0.07(0.05)$ & $0.00(0.06)$ & 0.95 & $-0.12(0.03)^{*}$ & $0.06(0.04)$ & 0.53 \\
\hline Ethical & $-0.04(0.03)$ & $-0.06(0.06)$ & $0.02(0.06)$ & 0.64 & $-0.13(0.03)^{*}$ & $0.09(0.04)^{*}$ & 0.28 \\
\hline
\end{tabular}

Notes: Relative effect sizes are the replication summary effect sizes divided by the ABP replication or original effect size: values less than 1 indicate summary effect size is smaller than the ABP or original effect size. Relative effect sizes are not calculated if replication estimates are the opposite sign of comparison estimates. $P<0.05^{*}$.

\section{A.9 Attitudes toward immigrants}

In the original study (Hainmueller and Hopkins, 2015), 1,407 U.S. respondents from a nationally representative online survey fielded by Knowledge Networks in 2012 participated in a conjoint experiment that asked them to choose between different pairs of hypothetical 
immigrants applying for admission. Each respondent evaluated five different pairs of immigrants, with immigrants' backgrounds varying along nine randomly assigned attributes: gender, education, employment plans, job experience, profession, language skills, country of origin, reasons for applying, and prior trips to the United States. Each attribute contained multiple levels (e.g. country was 10 levels and gender was 2) for a total of approximately 900,000 unique immigrant profiles.

After viewing each immigrant pair subjects were presented with a binary choice: "If you had to choose between them, which of these two immigrants should be given priority to come to the United States to live?" Each subject evaluated 5 pairs of immigrants for a total of 14,070 observations (1,407 respondents $\times 5$ pairs $\times 2$ immigrants per pair). We conducted a direct replication of this conjoint experiment in May 2020 on a sample of 1,328 respondents, for a total of 13,280 observations.

Following Hainmueller and Hopkins (2015), we estimate the Average Marginal Component Effects (AMCEs) for each attribute level using a regression of the binary response (1 if the immigrant profile is preferred, 0 otherwise) on a set of indicators for each attribute level, with standard errors clustered at the level of the survey respondent. Figure A.10 plots the results for the original study alongside our direct replication. The top of each panel describes the omitted reference level for each attribute; for example, the negative point estimates for "Male" indicate that male immigrants are about 2 percentage points less likely to be selected than female immigrants.

In total, there are 81 estimated AMCEs in Figure A.10 - 41 for the original study and 41 for the replication. When compared to the original study, the replication estimates are remarkably similar in both direction and magnitude. Only one of 41 replication estimates is signed in the opposite direction when compared to the original study - the AMCE for "Gardener" is 0.02 points in the original study and approximately zero in the replication, but neither estimate is distinguishable from zero. The majority of the replication estimates 
(27 of 41) are smaller in magnitude than the original estimates. ${ }^{4}$ We therefore conclude that the conjoint experiment was successfully replicated.

${ }^{4}$ only 7 of 41 differences are statistically significant after correcting for multiple comparisons to control the false discovery rate (see e.g. Benjamini and Hochberg, 1995). 


\section{Figure A.10: Effects of immigrant attributes on support for admission to U.S.}

Gender (reference: Female applicant)

Male

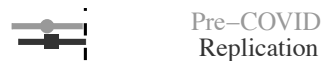

Education (reference: No formal education)

4th grade

8th grade

High school

Two-year college

College degree

Graduate degree

Broken English

Tried English but unable

Used interpreter

France

Mexico

Philippines

Poland

India

China

Sudan

Somalia

Iraq

Profession (reference: Janitor)

Waiter

Child care provider

Gardener

Financial analyst

Construction worker

Teacher

Computer programmer

Nurse

Research scientist

Doctor

$1-2$ years
$3-5$ years
$5+$ years

Job experience (reference: No job training or prior experience)

Interviews with employer

Will look for work

No plans to look for work

Seek better job

Escape persecution

Application reason (reference: Reunite with family members already in the U.S.)

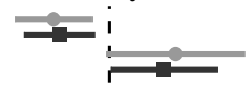

Prior trips to U.S. (reference: Never been to the U.S.)

Once as tourist

Many times as tourist

Six months with family

Once w/o authorization

Jobs plans (reference: Contract with a U.S. employer)
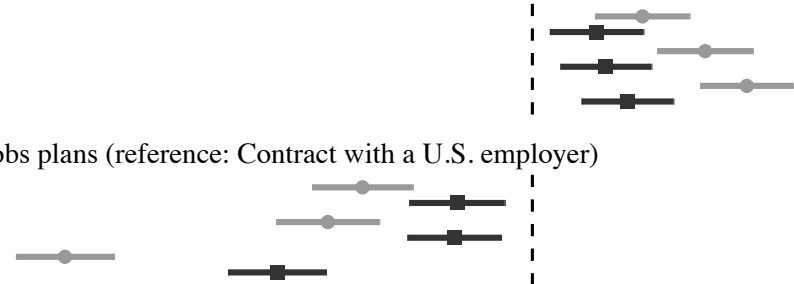

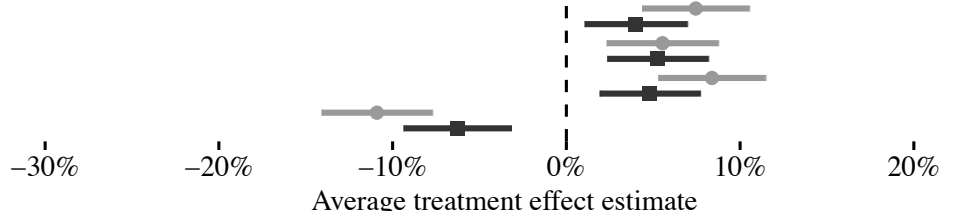




\section{A.10 Fake news corrections}

In the original study (Porter, Wood and Kirby, 2018), 2,742 MTurk workers were exposed to two fake news stories randomly selected from a sample of six fake news stories that were previously circulated (e.g. that Obama's birth certificate is fake). For each fake news story, subjects were randomly assigned to see either a correction following the story, or no correction. Therefore subjects in the "correction" (treatment) condition read a randomly assigned story followed by a correction stating that the story was false, whereas subjects in the "no correct" (control) condition simply read the story without seeing a correction. Therefore, the experiment has $6 \times 2=12$ treatment arms, with each respondent being exposed to two unique stories with or without a correction.

The goal of this study was to test whether corrections could reduce individuals' beliefs in the veracity of fake news stories. Following exposure to the fake news story (and the correction if assigned treatment) subjects were asked to indicate their agreement or disagreement about the truth value of the claim advanced on a 5-point scale. Across all six fake news stories, the authors found that exposure to corrections caused a significant reduction in respondents' beliefs that the stories were true, with average treatment effects ranging from -0.24 scale points on the low end to -0.95 scale points on the high end.

We conducted a direct replication of this experiment on a sample of 1,415 respondents in April 2020. Following the original study, we scale outcomes so that higher values indicate stronger agreement that the fake news stories were true. Within randomly assigned story, outcomes are standardized by dividing the response vector by the standard deviation in the "no correction" (control) group. Figure A.11 compares estimated treatment effects between the original study and replication, for each of the six stories. All replication estimates, ranging from -0.04 to -0.67 , are uniformly smaller then those in the original study, but all are correctly signed. ${ }^{5}$ We therefore conclude that that the replication was successful.

\footnotetext{
${ }^{5}$ Although all 6 replication estimates are smaller in magnitude than those in the original study, only two
} 
Figure A.11: Effect of corrections on agreement with inaccurate statements

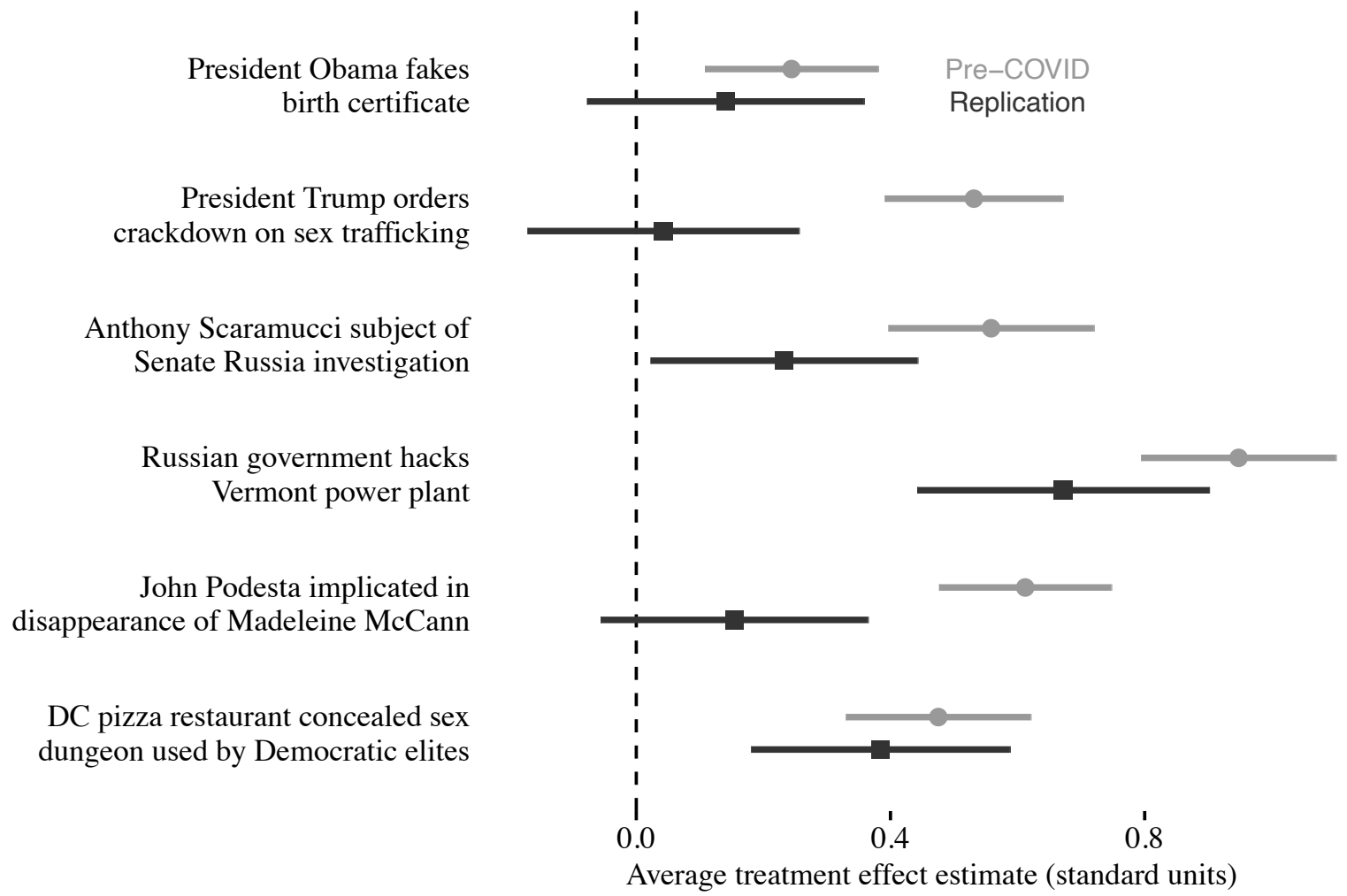

\section{A.11 Inequality and System Justification}

In the original study (Trump and White, 2018), 1,020 U.S. respondents from a nationally representative online survey fielded by Knowledge Networks in 2015 participated in a survey experiment with random assignment to two conditions. In the "low-inequality" (control) condition, respondents were exposed to information about trends in U.S. income inequality, as measured by the Gini coefficient over the period 1968-2010. In the "high-inequality" (treatment) condition, respondents were exposed to the same information, but the y-axis in the plot was truncated to make the upward trend appear much steeper.

The goal of this study was the test a hypothesis that exposure to inequality increases "system justification" - broadly, the psychological need to support the status quo, even at

of these differences (John Podesta and Trump stories) are statistically distinguishable from zero. 
the expense of their self-interest, or the interests of their group (see e.g. Jost and Banaji, 1994). Trump and White (2018) test the hypothesis that higher inequality causes higher system justification by comparing differences in subjects' system justification scores between the high and low inequality conditions. The key prediction is that increasing subjects' beliefs that inequality is rising should decrease system justification.

Following exposure to one of the two data visualizations, respondents completed two "manipulation check" questions. For the first, respondents were asked to say whether the statement "Income inequality in the United States has increased dramatically over time" was correct or incorrect. The second asked respondents whether the statement "the share of total income of the very rich has not changed much over time in the United States" was correct or incorrect. According to Trump and White (2018), the high-inequality treatment should cause an increase in the proportion of respondents stating "correct" to the first question and "incorrect" to the second question, relative to control. Responses to the first question are therefore coded 1 if the subject answers "correct" and 0 otherwise. Responses to the second questions are coded 1 if the subject answers "incorrect" and 0 otherwise.

After this, respondents were randomly assigned to complete one of three batteries of questions the authors used to measure system justification: an institutional trust scale (6items), a system justification scale (8-items), or an economic system justification scale (15items). In total, 339 subjects (169 in control, 170 in treatment) completed the system justification scale, 338 completed the institutional trust scale (169 in treatment, 169 in control), and 336 completed the economic system justification scale (167 in treatment and 169 in control). In our replication, 804 subjects were presented with all three system justification scales in randomized order. Following the original study, we scale outcomes so that higher values indicate higher levels of system justification.

Figure A.12 compares estimated treatment effects on the manipulation check questions (top panel) and outcomes scales (bottom panel) between the original study and the replica- 
tion. All replication estimates are in the expected direction when compared to the original study. Although all 5 replication estimates are smaller in magnitude than those in the original study, none of these differences are statistically distinguishable from zero. We therefore conclude that the replication was successful.

Figure A.12: Effect of "high inequality" treatment on comprehension questions and system justification scales

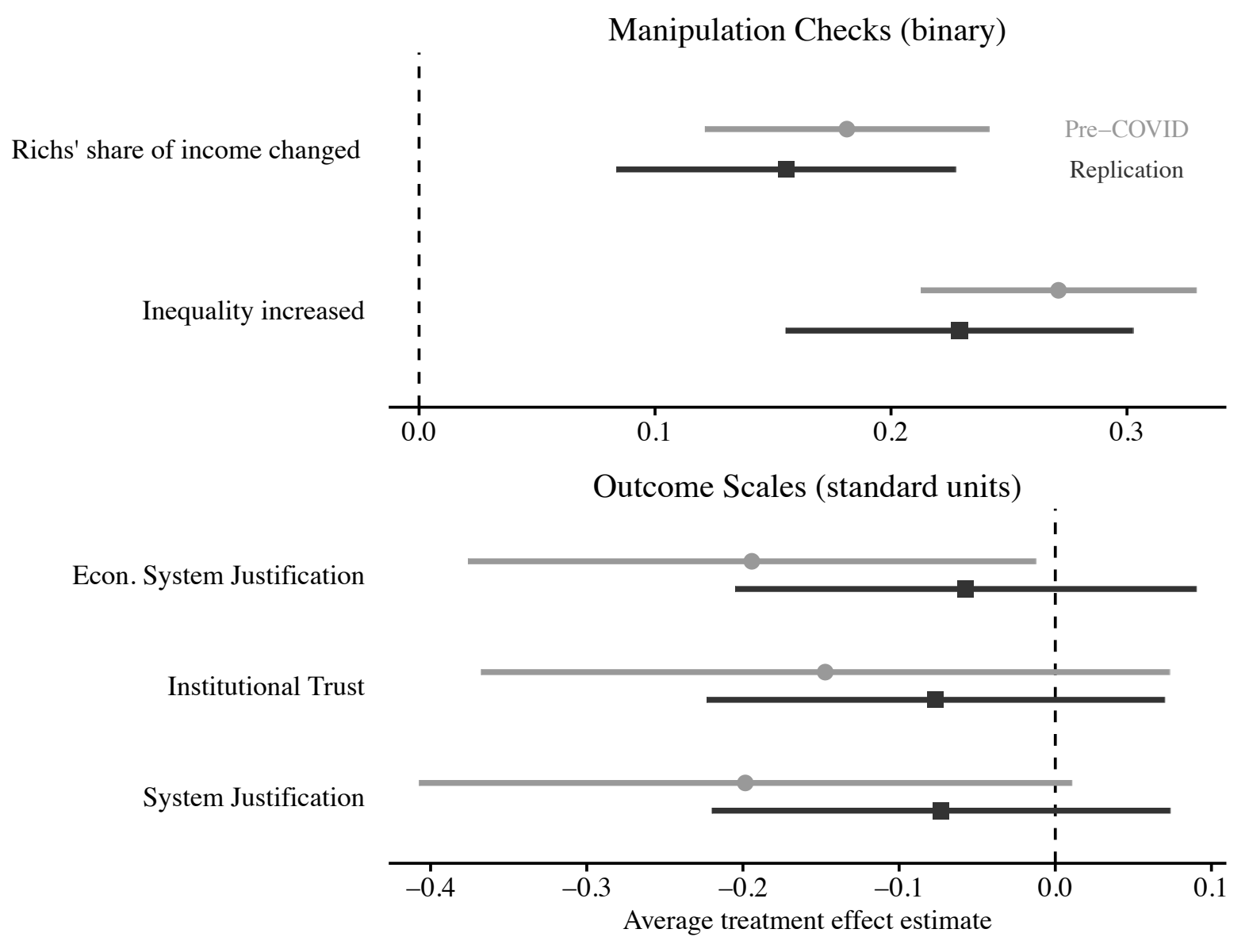

\section{A.12 Trust in government and redistribution}

In the original study (Peyton, 2020), a total of 3,837 U.S. respondents were exposed to information about corruption in American government across three separate experiments: Experiment 1 (624 MTurk workers in 2014); Experiment 2 (nationally representative sample 
of 1,324 U.S. adults in 2014); Experiment 3 (1,870 MTurk workers in 2017). In each experiment, participants were randomly assigned to one of three treatment arms: "Corrupt", "Honest", or "Control". In the "Corrupt" arm, subjects read an Op-Ed by a former DOJ prosecutor that described high levels of political corruption in American politics; the "Honest" arm used contrasting language to describe low levels of political corruption. In the "Control" arm, subjects read an article of similar length that was devoid of political content. Experiment 2 was a direct replication of Experiment 1, and Experiment 3 supplemented the articles with data visualizations that supported the writers' arguments.

These experiments were used to test a theory that increasing trust in government causes Americans to become more supportive of redistribution. Peyton (2020) tests this by experimentally manipulating respondents' trust in government and testing for downstream effects on respondent's support for redistribution using a causal instrumental variables framework. Following exposure to treatment, subjects' trust in government was measured using a 4item scale. Next, subject's support for redistribution was measured using a 4-item scale about federal spending redistributive social policies. The author found significant effects on subjects' trust in government in all three experiments, but support for redistribution was indistinguishable from zero.

We conducted a direct replication of Experiment 3 in the original study on a sample of 1,424 respondents in May 2020. Following the original study, treatment was coded 0 if a subject was assigned to the "Corrupt" arm, 0.5 if assigned "Control", and 1 if assigned "Honest". Outcomes were scaled so that higher values indicate more trust in government, and more support for redistribution.

Figure A.13 compares estimated treatment effects on trust in government (top panel) and support for redistribution (bottom panel) between the replication and Experiments 1-3 in the original study. The estimated treatment effect on trust in government in the replication is statistically distinguishable from zero, and in the expected direction. However, this estimate 
is significantly smaller in magnitude than all of the estimates in the original study. The estimated treatment effects on support for redistribution are indistinguishable from zero in the replication, and statistically indistinguishable from the estimates in the original study. We therefore conclude this was a successful replication.

Figure A.13: Effect of corruption information on trust in government and support for redistribution

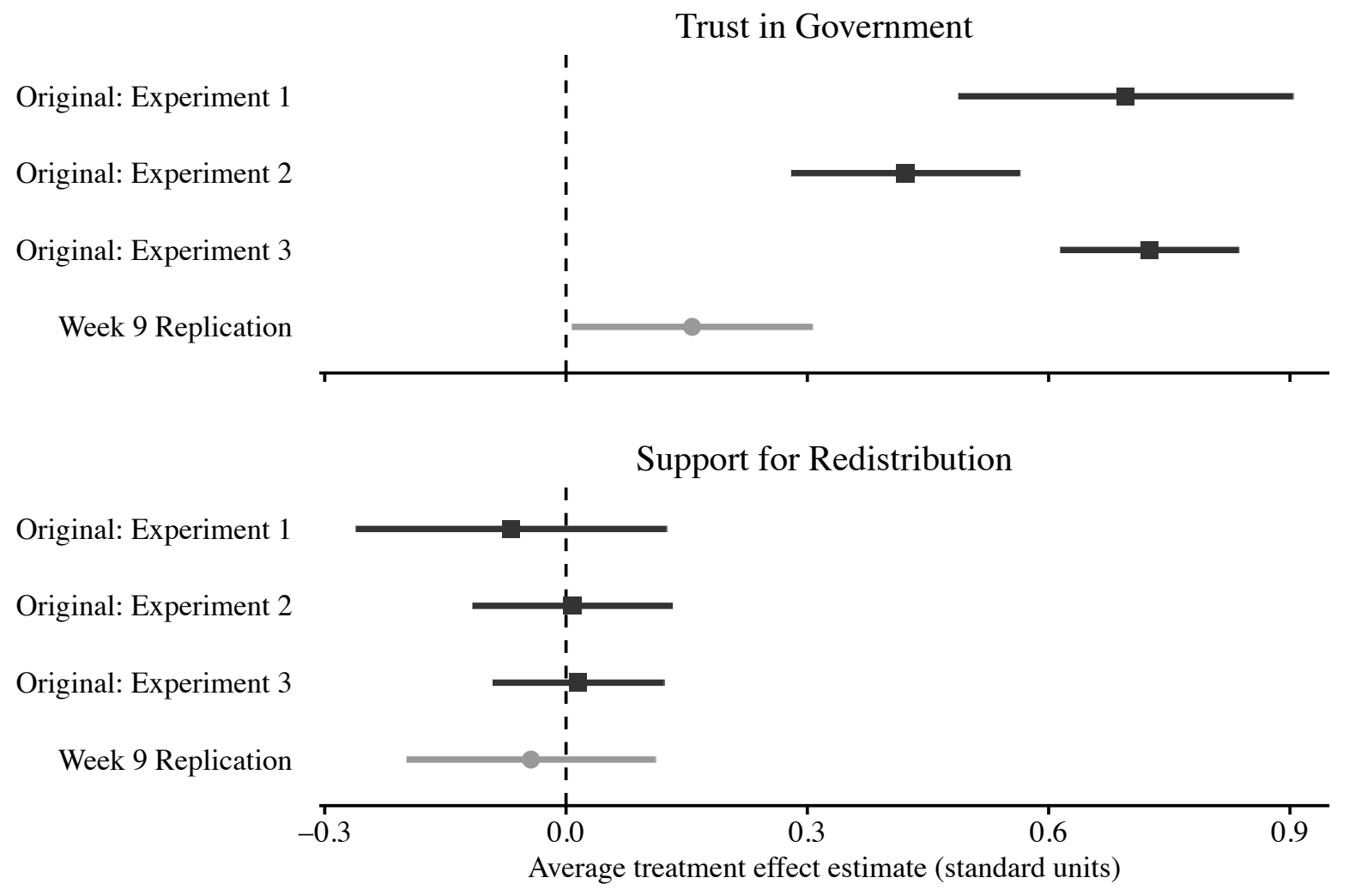




\section{B Covariate distributions}

In the manuscript, we note that attention check questions (ACQs) can be used to either screen out inattentive respondents at the design stage, or to estimate treatment effects among the subset of attentive participants at the analysis stage. We caution, however, that any procedure for measuring inattention is itself an estimator for a latent individual-level characteristic (i.e., "attentiveness"). As Berinsky, Margolis and Sances (2014) have shown, respondents that fail attention checks can differ markedly from those who pass on observed characteristics. We find that classifiers based on respondents' metadata are correlated with some covariates as well, suggesting that survey designs that restrict participation to respondents from non-mobile devices or internet browsers could also have implications for sample composition. These estimates are presented below for the pooled sample of respondents across all 13 surveys that we conducted between March and July 2020.

Figure B.1: Region proportions by sample

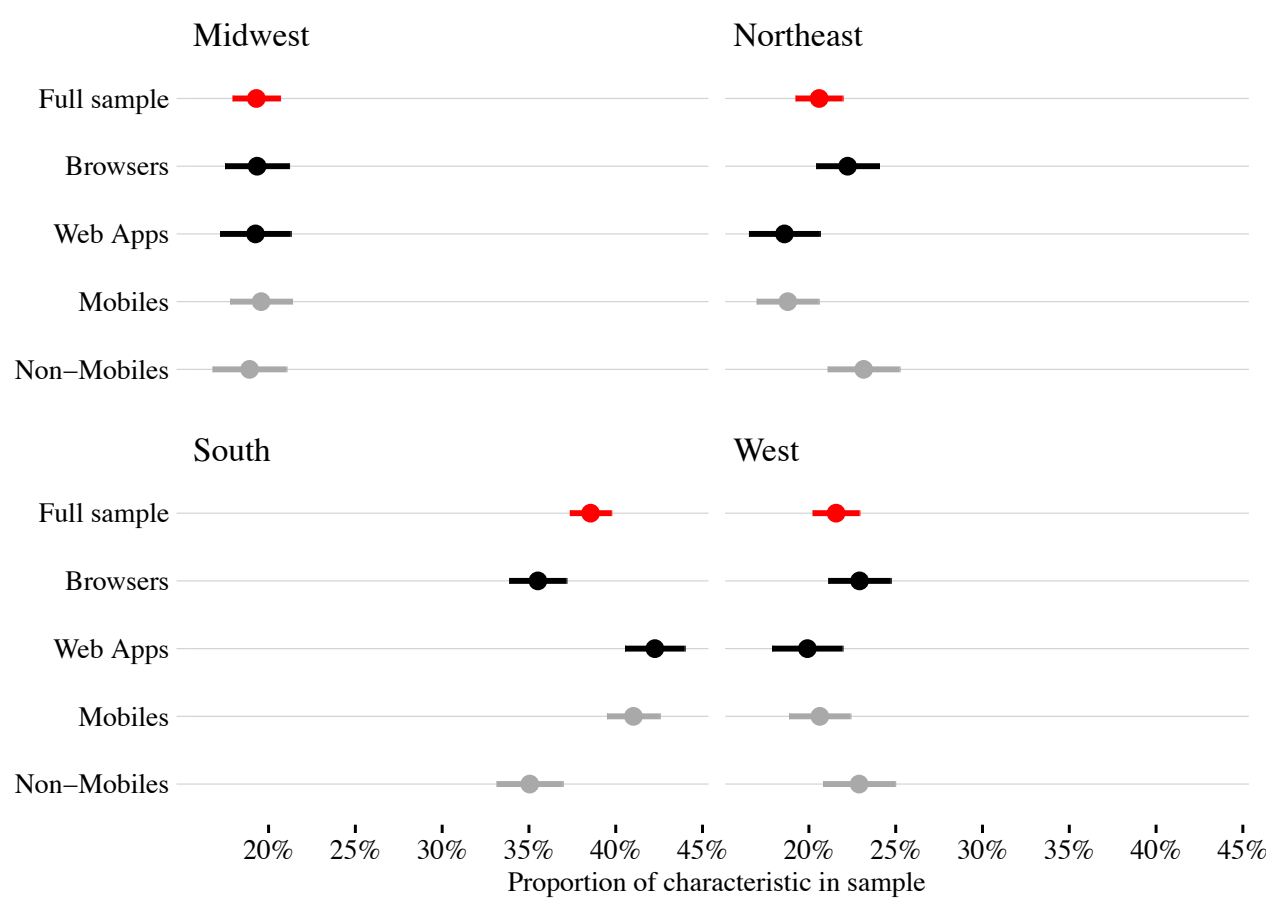


Figure B.2: Education proportions by sample

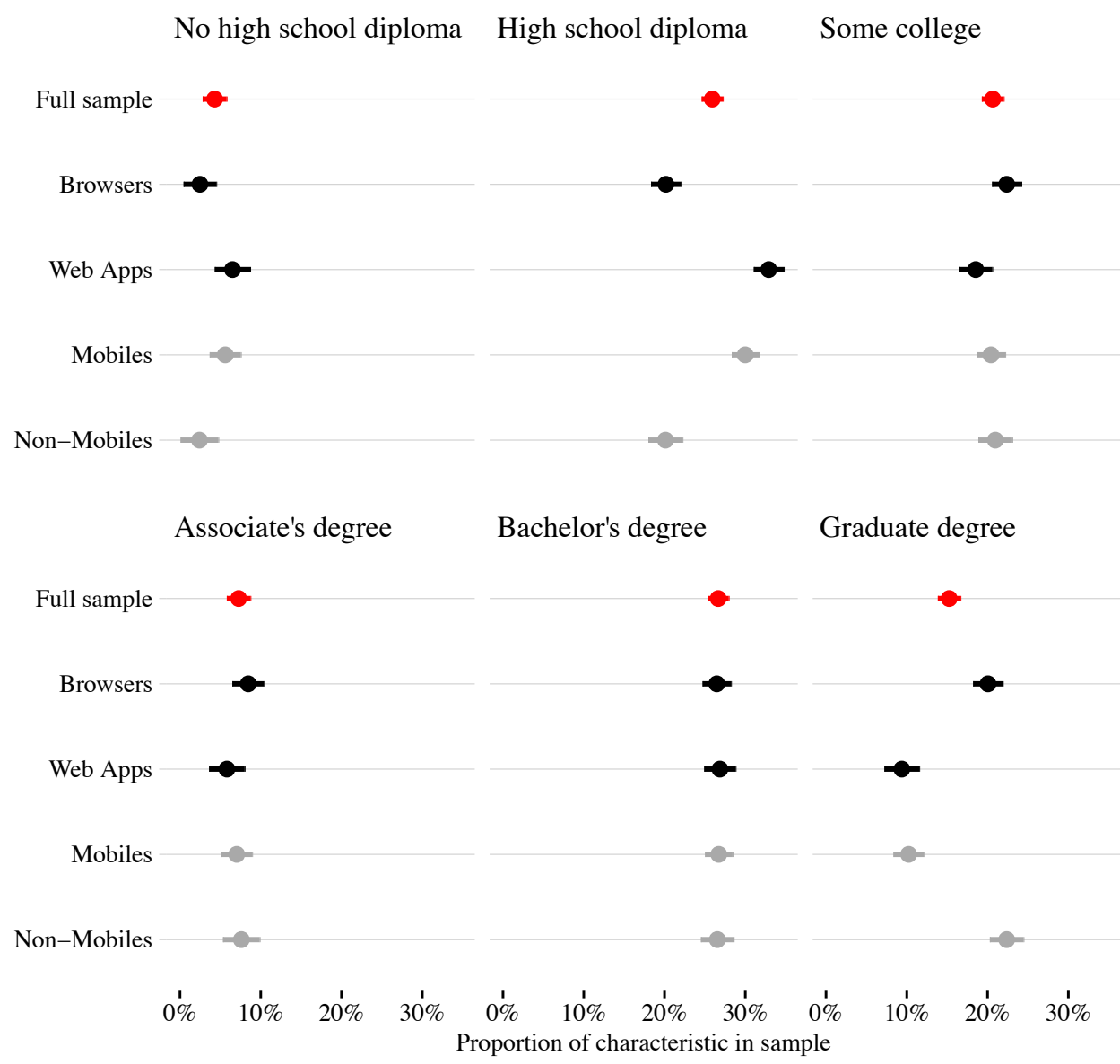


Figure B.3: Household income proportions by sample

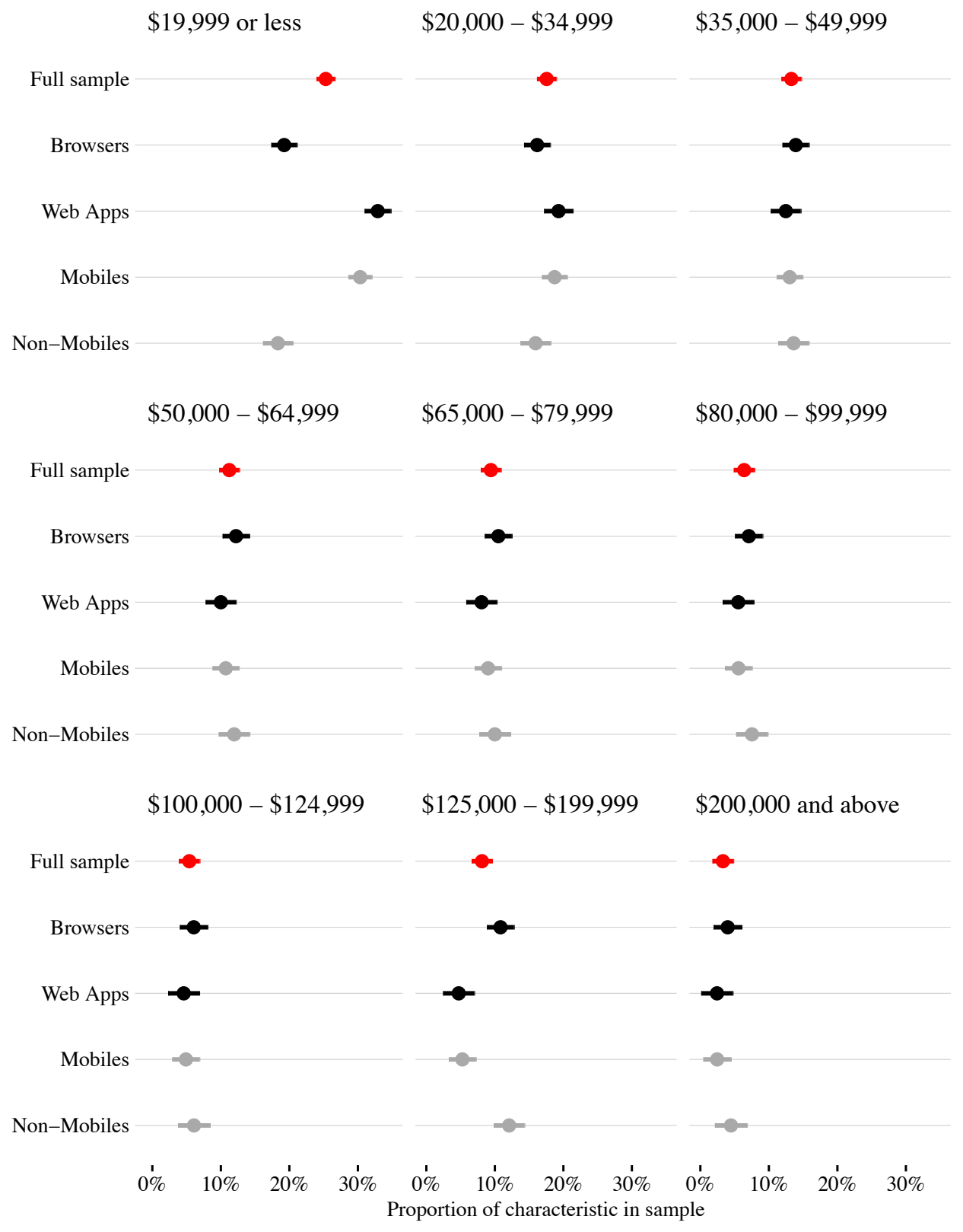


Figure B.4: Age proportions by sample

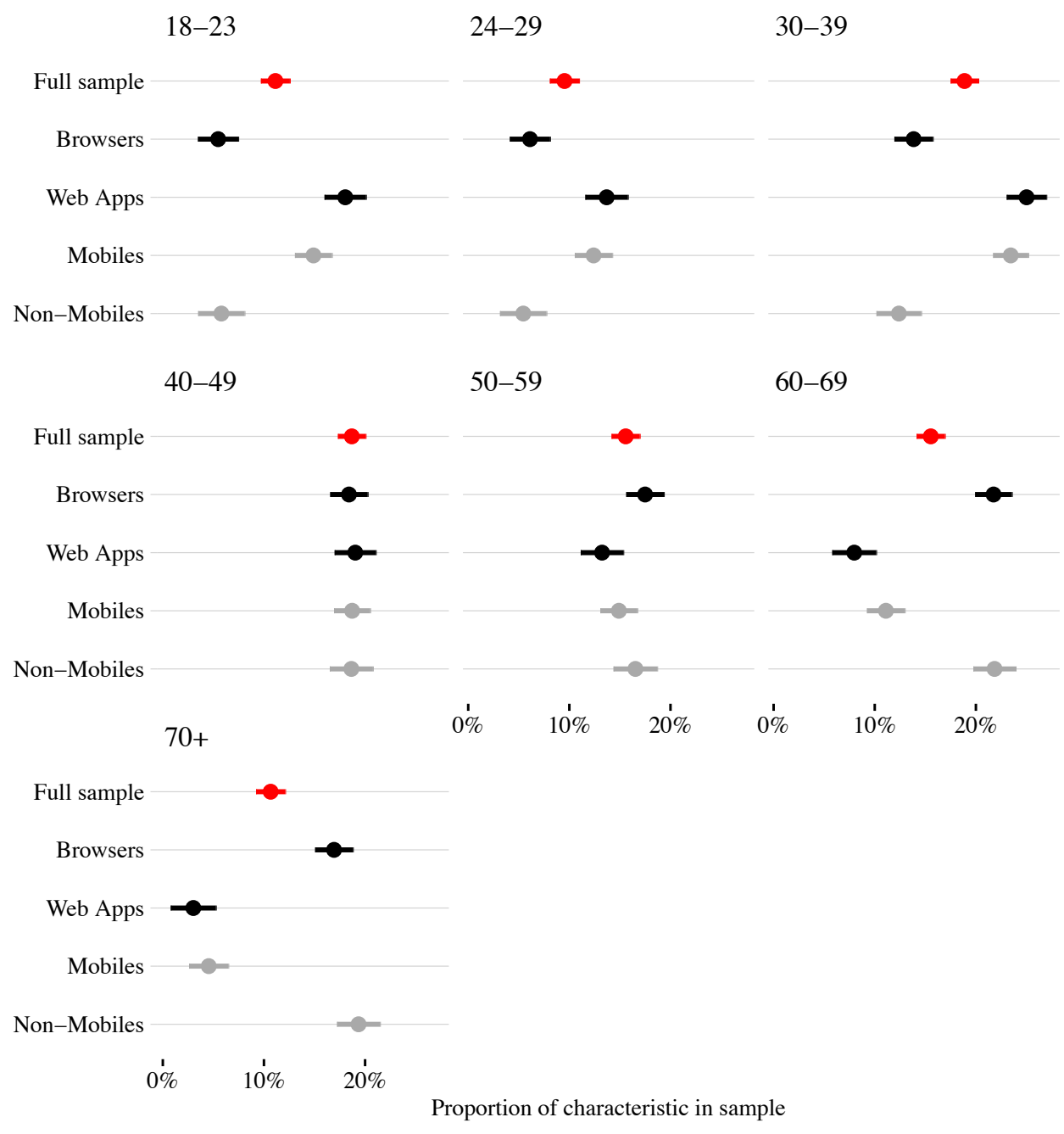


Figure B.5: Male v. Female proportions by sample

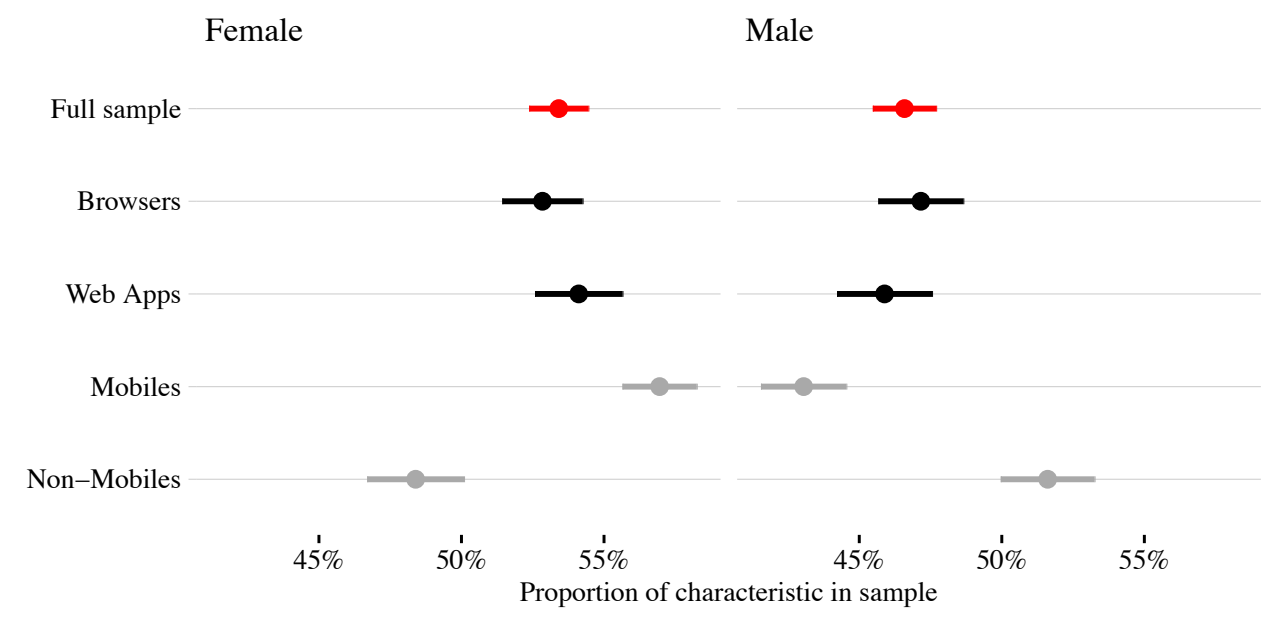

Figure B.6: Race/Ethnicity proportions by sample

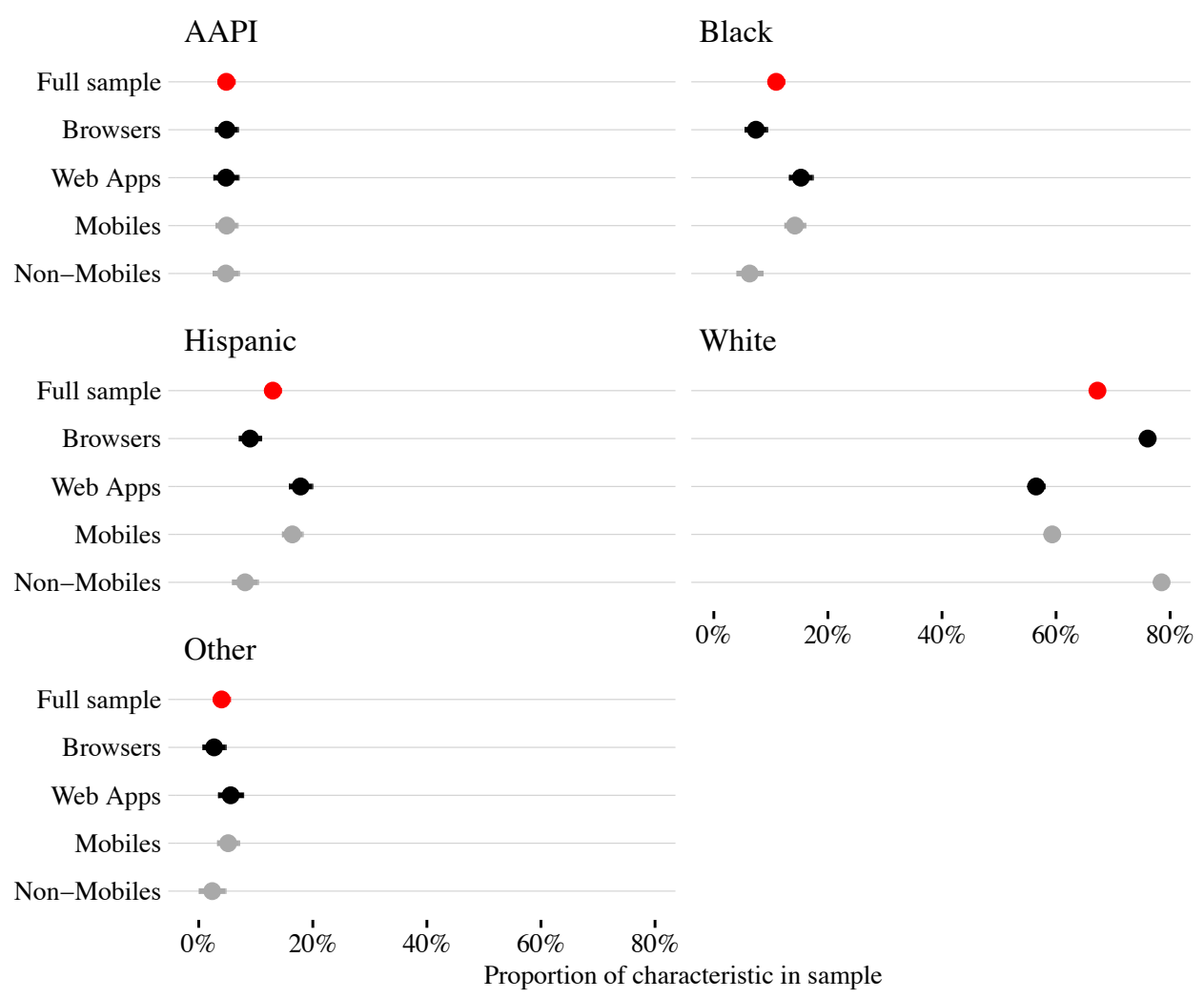


Figure B.7: Partisanship proportions by sample

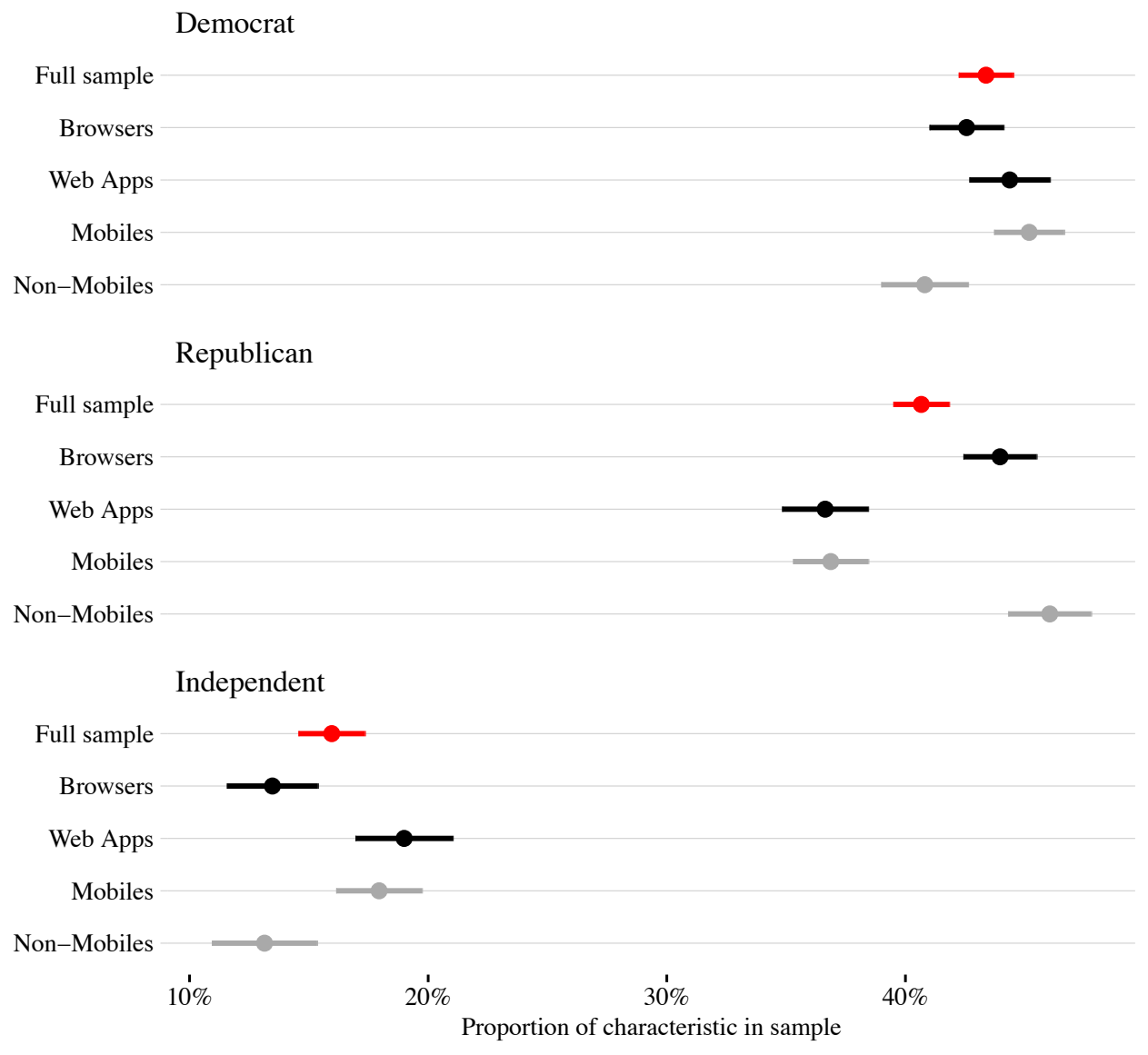


Figure B.8: Voting behavior in 2016 proportions by sample

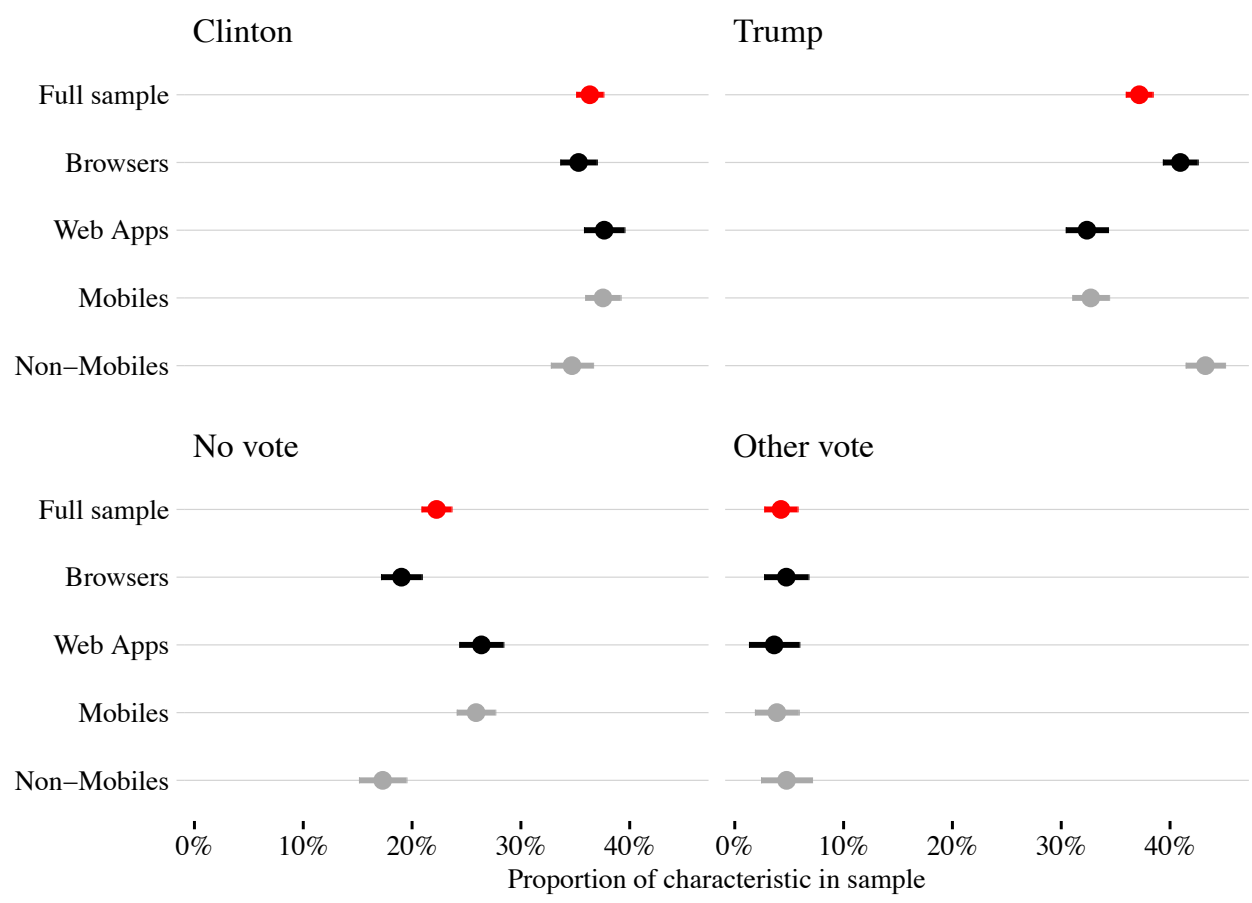




\section{Treatment descriptions}

Figure C.1: Effect of framing on decision making: cheap condition (original)

Imagine that you are about to purchase a ceramic vase for $\$ 250$, and a wall hanging for $\$ 30$. The salesman informs you that the wall hanging you wish to buy is on sale for $\$ 20$ at the other branch of the store, located 20 minutes drive away. Would you make the trip to the other store?

Yes, I would go to the other branch

No, I would not go to the other branch

Figure C.2: Effect of framing on decision making: expensive condition (original) Imagine that you are about to purchase a ceramic vase for $\$ 30$, and a wall hanging for $\$ 250$. The salesman informs you that the wall hanging you wish to buy is on sale for $\$ 240$ at the other branch of the store, located 20 minutes drive away. Would you make the trip to the other store?

Yes, I would go to the other branch

No, I would not go to the other branch

Figure C.3: Effect of framing on decision making: cheap condition (modified)

Imagine that you are about to purchase a large box of Clorox disinfecting wipes for $\$ 250$, and a large box of $\mathrm{N}-95$ respirator masks for $\$ 30$. The salesman informs you that the box of respirator masks you wish to buy is on sale for $\$ 20$ at the other branch of the store, located 20 minutes drive away. Would you make the trip to the other store?

Yes, I would go to the other branch

No, I would not go to the other branch

Figure C.4: Effect of framing on decision making: expensive condition (modified) Imagine that you are about to purchase a large box of Clorox disinfecting wipes for $\$ 30$, and a large box of $\mathrm{N}-95$ respirator masks for $\$ 250$. The salesman informs you that the box of respirator masks you wish to buy is on sale for $\$ 240$ at the other branch of the store, located 20 minutes drive away. Would you make the trip to the other store?

Yes, I would go to the other branch

No, I would not go to the other branch 
Figure C.5: Perceived intentionality for side effects: helped condition (original)

The vice-president of a company went to the chairman of the board and said, "We are thinking of starting a new program. It will help us increase profits, and it will also help the environment."

The chairman of the board answered, "I don't care at all about helping the environment. I just want to make as much profit as I can. Let's start the new program."

They started the new program. Sure enough, the environment was helped.

How much do you agree with the statement: "The chairman helped the environment intentionally."
Strongly agree
Agree
Somewhat agree
Neither agree nor disagree
Somewhat disagree
$\mathrm{O}$ Disagree
Strongly disagree 
Figure C.6: Perceived intentionality for side effects: harmed condition (original)

The vice-president of a company went to the chairman of the board and said, "We are thinking of starting a new program. It will help us increase profits, but it will also harm the environment."

The chairman of the board answered, "I don't care at all about harming the environment. I just want to make as much profit as I can. Let's start the new program."

They started the new program. Sure enough, the environment was harmed.

How much do you agree with the statement: "The chairman harmed the environment intentionally."

Strongly agree

$\bigcirc$ Agree

Somewhat agree

Neither agree nor disagree

Somewhat disagree

Disagree

Strongly disagree 
Figure C.7: Perceived intentionality for side effects: helped condition (modified)

The vice-president of a company went to the chairman of the board and said, "We are thinking of marketing a new drug to treat COVID-19. It will help us increase profits, and the drug will also help older people with heart conditions."

The chairman of the board answered, "I don't care at all about helping older people with heart conditions. I just want to make as much profit as I can. Let's start marketing the new drug."

They started marketing the new drug. Sure enough, older people with heart conditions were helped.

How much do you agree with the statement: "The chairman helped older people with heart conditions intentionally."
Strongly agree
$\bigcirc$ Agree
Somewhat agree
Neither agree nor disagree
Somewhat disagree
Disagree
Strongly disagree 
Figure C.8: Perceived intentionality for side effects: harmed condition (modified)

The vice-president of a company went to the chairman of the board and said, "We are thinking of marketing a new drug to treat COVID-19. It will help us increase profits, but the drug will also harm older people with heart conditions."

The chairman of the board answered, "I don't care at all about harming older people with heart conditions. I just want to make as much profit as I can. Let's start marketing the new drug."

They started marketing the new drug. Sure enough, older people with heart conditions were harmed.

How much do you agree with the statement: "The chairman harmed older people with heart conditions intentionally."
Strongly agree
Agree
Somewhat agree
Neither agree nor disagree
Somewhat disagree
$\bigcirc$ Disagree
Strongly disagree 


\section{C.1 Attention Check Questions}

Figure C.9: Pre-ACQ article for "Easy" and "Medium" ACQ MAN ARRESTED FOR STRING OF BANK THEFTS

Columbus Police have arrested a man they say gave his driver's license to a teller at a bank he was robbing.

According to court documents, Bryan Simon is accused of robbing four Central Ohio banks between October 3 and November 5, 2018.

During a robbery on November 5 at the Huntington Bank, the sheriff's office says Simon was tricked into giving the teller his drivers' license.

According to court documents, Simon approached the counter and presented a demand note for money that said "I have a gun." The teller gave Simon about $\$ 500$, which he took.

Documents say Simon then told the teller he wanted more money. The teller told him a driver's license was required to use the machine to get our more cash. Simon reportedly then gave the teller his license to swipe through the machine and then left the bank with about $\$ 1000$ in additional cash, but without his ID.

Detectives arrested him later that day at the address listed on his ID. 
Figure C.10: "Easy" and "Medium" ACQ with correct responses highlighted How was Simon identified by police for the crime he allegedly committed?

A police officer recognized him

From video surveillance

Because he left his ID

He turned himself in

None of the above

How much money did Simon allegedly steal?

About $\$ 500$

About $\$ 1500$

About $\$ 25,000$

About $\$ 1$ million dollars

$\mathrm{O}$ None of the above

Figure C.11: "Hard" ACQ with correct response highlighted In this prompt we are going to ask you to answer a question about mathematics. Although not everyone likes mathematics we believe this is a relatively simple question to answer. Having said that, we would like you to answer eight regardless of what you think the correct answer is.

Please solve the following math problem: What is $(2+2) / 1=?$

$\begin{array}{ll}\mathrm{O}_{8} & \mathrm{O}_{6} \\ \mathrm{O}_{4} & \mathrm{O}_{2}\end{array}$




\section{References}

Aronow, Peter M, Jonathon Baron and Lauren Pinson. 2019. "A note on dropping experimental subjects who fail a manipulation check." Political Analysis 27(4):572-589.

Benjamini, Yoav and Yosef Hochberg. 1995. "Controlling the false discovery rate: a practical and powerful approach to multiple testing." Journal of the Royal statistical society: series $B$ (Methodological) 57(1):289-300.

Berinsky, Adam J, Gregory A Huber and Gabriel S Lenz. 2012. "Evaluating online labor markets for experimental research: Amazon.com's Mechanical Turk." Political analysis $20(3): 351-368$.

Berinsky, Adam J, Michele F Margolis and Michael W Sances. 2014. "Separating the shirkers from the workers? Making sure respondents pay attention on self-administered surveys." American Journal of Political Science 58(3):739-753.

Clifford, Scott, Geoffrey Sheagley and Spencer Piston. 2020. "Increasing Precision in Survey Experiments Without Introducing Bias." Unpublished Manuscript

Coppock, Alexander and Oliver A McClellan. 2019. "Validating the demographic, political, psychological, and experimental results obtained from a new source of online survey respondents." Research \& Politics 6(1):2053168018822174.

Druckman, James N. 2001. "Using credible advice to overcome framing effects." Journal of Law, Economics, and Organization 17(1):62-82.

Gilens, Martin. 2001. "Political ignorance and collective policy preferences." American Political Science Review pp. 379-396.

Glass, Gene V. 1976. "Primary, secondary, and meta-analysis of research." Educational researcher $5(10): 3-8$. 
Hainmueller, Jens and Daniel J Hopkins. 2015. "The hidden American immigration consensus: A conjoint analysis of attitudes toward immigrants." American Journal of Political Science 59(3):529-548.

Huber, Gregory A and Celia Paris. 2013. "Assessing the programmatic equivalence assumption in question wording experiments: Understanding why Americans like assistance to the poor more than welfare." Public Opinion Quarterly 77(1):385-397.

Hyman, Herbert H and Paul B Sheatsley. 1950. "The Current Status of American public opinion." The Teaching of Contemporary Affairs pp. 11-34.

Jost, John T and Mahzarin R Banaji. 1994. "The role of stereotyping in system-justification and the production of false consciousness." British journal of social psychology 33(1):1-27.

Klein, Richard A, Kate A Ratliff, Michelangelo Vianello, Reginald B Adams Jr, Štěpán Bahník, Michael J Bernstein, Konrad Bocian, Mark J Brandt, Beach Brooks, Claudia Chloe Brumbaugh et al. 2014. "Investigating variation in replicability." Social psychology .

Klein, Richard A, Michelangelo Vianello, Fred Hasselman, Byron G Adams, Reginald B Adams Jr, Sinan Alper, Mark Aveyard, Jordan R Axt, Mayowa T Babalola, Štěpán Bahník et al. 2018. "Many Labs 2: Investigating variation in replicability across samples and settings." Advances in Methods and Practices in Psychological Science 1(4):443-490.

Knobe, Joshua. 2003. "Intentional action and side effects in ordinary language." Analysis 63(3):190-194.

Peyton, Kyle. 2020. "Does Trust in Government Increase Support for Redistribution? Evidence from Randomized Survey Experiments." American Political Science Review 114(2):596-602. 
Porter, Ethan, Thomas J Wood and David Kirby. 2018. "Sex trafficking, Russian infiltration, birth certificates, and pedophilia: A survey experiment correcting fake news." Journal of Experimental Political Science 5(2):159-164.

Press, Daryl G, Scott D Sagan and Benjamin A Valentino. 2013. "Atomic aversion: Experimental evidence on taboos, traditions, and the non-use of nuclear weapons." American Political Science Review pp. 188-206.

Schuman, Howard and Stanley Presser. 1996. Questions and answers in attitude surveys: Experiments on question form, wording, and context. Sage.

Smith, Tom W. 1987. "That which we call welfare by any other name would smell sweeter an analysis of the impact of question wording on response patterns." Public opinion quarterly $51(1): 75-83$.

Trump, Kris-Stella and Ariel White. 2018. "Does inequality beget inequality? Experimental tests of the prediction that inequality increases system justification motivation." Journal of Experimental Political Science 5(3):206-216.

Tversky, Amos and Daniel Kahneman. 1981. "The framing of decisions and the psychology of choice." science 211(4481):453-458. 